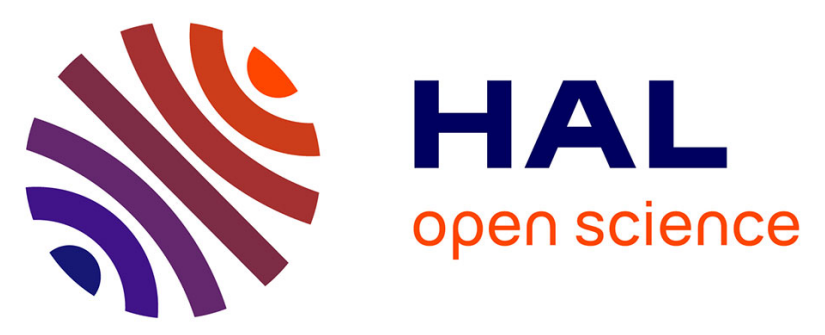

\title{
Syntheses and kinetic studies of cyclisation-based self-immolative spacers
}

Steve Huvelle, Ahmed Alouane, Thomas Le Saux, Ludovic Jullien, Frédéric Schmidt

\section{- To cite this version:}

Steve Huvelle, Ahmed Alouane, Thomas Le Saux, Ludovic Jullien, Frédéric Schmidt. Syntheses and kinetic studies of cyclisation-based self-immolative spacers. Organic \& Biomolecular Chemistry, 2017, 15 (16), pp.3435 - 3443. 10.1039/C7OB00121E . hal-01513562

\section{HAL Id: hal-01513562 https://hal.sorbonne-universite.fr/hal-01513562}

Submitted on 25 Apr 2017

HAL is a multi-disciplinary open access archive for the deposit and dissemination of scientific research documents, whether they are published or not. The documents may come from teaching and research institutions in France or abroad, or from public or private research centers.
L'archive ouverte pluridisciplinaire HAL, est destinée au dépôt et à la diffusion de documents scientifiques de niveau recherche, publiés ou non, émanant des établissements d'enseignement et de recherche français ou étrangers, des laboratoires publics ou privés. 


\section{Organic \& Biomolecular Chemistry}

\section{PAPER}

Cite this: Org. Biomol. Chem., 2017. 15, 3435

Received 17th January 2017

Accepted 21st March 2017

DOI: $10.1039 / \mathrm{c} 7 \mathrm{ob} 00121 \mathrm{e}$

\section{Syntheses and kinetic studies of cyclisation-based self-immolative spacers $\uparrow$}

\author{
Steve Huvelle, ${ }^{a}$ Ahmed Alouane, ${ }^{a, b, c, d}$ Thomas Le Saux, ${ }^{b, c, d}$ Ludovic Jullien ${ }^{\star b, c, d}$ and \\ Frédéric Schmidt (D) *a
}

Kinetic analysis of the disassembly of self-immolative spacers based on cyclisation processes was performed. Five compounds were synthesized belonging to two different series, and their kinetic constants were determined. Electron-donating substituents gave a slight acceleration but the main effect was steric, and the Thorpe-Ingold effect was indeed particularly effective. Comparison with the self-immolative spacers based on elimination processes showed that cyclisations gave comparable or lower rate, but the corresponding spacers are more difficult to modulate.

\section{Introduction}

Self-immolative spacers were introduced in 1981 by Katzellenbogen et al. ${ }^{1}$ as an original way to correlate the cleavage of two chemical bonds. In a medicinal chemistry context, this strategy was proposed to overcome limitations of prodrugs, classically made of two moieties: an activator, reacting with an enzyme as a substrate, and a bioactive compound, as a drug or a reporter. The introduction of the spacer core involves the addition of a third moiety designed to release the effector after activation. The second bond is cleaved spontaneously after cleavage of the trigger (Scheme 1). A variety of self-immolative spacers have been introduced over the years in the literature $^{2-4}$ and for instance used in cancer chemotherapy (Adcetris $\left.{ }^{\circledR}\right) .{ }^{5-8}$ In this strategy, the spontaneous release introduces a second step with its own kinetic parameters. For most applications (medicinal chemistry, analytical chemistry, materials or chemical biology), the self-immolative step needs to be fast enough in order to avoid the release too far from the activation site. Our aim has been to obtain comparative data on the kinetics of the self-immolative step so that anyone can choose a spacer suited to their applications. Kinetics of some self-immolative spacers have been studied during the past,

\footnotetext{
${ }^{a}$ Institut Curie, PSL Research University, CNRS UMR3666, INSERM U1143, 26 rue d'Ulm, F-75005 Paris, France. E-mail: Frederic.Schmidt@curie.fr ${ }^{b}$ École Normale Supérieure, PSL Research University, UPMC Univ Paris 06, CNRS, Département de Chimie, PASTEUR, 24 rue Lhomond, 75005 Paris, France. E-mail: Ludovic.Jullien@ens.fr

${ }^{c}$ Ecole Normale Supérieure, Département de Chimie, UMR CNRS ENS-UPMC 8640 PASTEUR24 rue Lhomond, 75231 Paris, France

${ }^{d}$ Sorbonne Universités, UPMC Univ Paris 06, ENS, CNRS, PASTEUR, 75005 Paris, France

$\dagger$ Electronic supplementary information (ESI) available. See DOI: 10.1039/ c7ob00121e
}

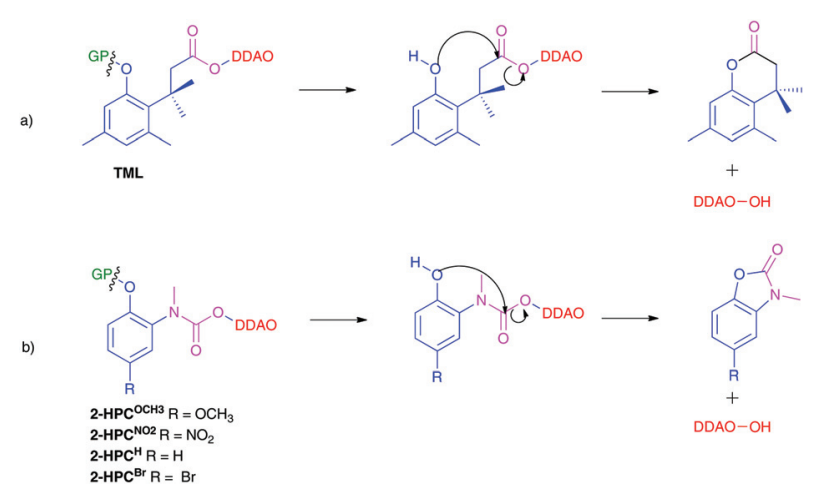

Scheme 1 Cyclisation-based spacers investigated, with (a) trimethyl lock and (b) 2-HPC. PG = 4,5-dimethoxy-2-nitrobenzyl; DDAO = 1,3dichloro-9,9-dimethyl-9H-acridin-2(7)-one.

especially for elimination-based releases. ${ }^{9-12}$ This is why we focused our present studies on the other class of spacers exploiting cyclisation in a self-immolative step. In this series of spacers, the activation step generates a nucleophilic heteroatom (like nitrogen or oxygen; less often sulfur ${ }^{13}$ ) which attacks an electrophilic centre (classically a carbonyl) on another position of the skeleton of the molecule ${ }^{14}$ (Scheme 1).

To measure accurately the kinetics of the self-immolation step, a general procedure was set up based on fast and controlled photoactivation; the fluorescence measurement of a reporter then gave access to the kinetic constants. ${ }^{9-12}$ We have already published comparative data on kinetic constants of various elimination-based spacers $(1,4$ and 1,6$)$ supported by aromatic $^{10,11}$ or heteroaromatic rings. ${ }^{12}$ In order to complete our comprehension of rate description of self-immolative spacers, we measured the kinetics of cyclisation-based spacers. Two main questions were addressed, the effect of the nature of 
the spacer and the comparison between the elimination and cyclisation processes.

\section{Results and discussion}

\section{Molecular design}

The structure of the targeted compounds was tripartite: a photocleavable moiety, the self-immolative spacer, and a reporter (fluorophore).

A classical 4,5-dimethoxy-2-nitrobenzyl (also called 6-nitroveratryl) group was used for the photocleavage step. This protecting group (denoted PG in Scheme 1) has been reported to be selectively cleaved by near-UV irradiation $(365 \mathrm{~nm})$, with excellent yield, and allows a millisecond timescale resolution. ${ }^{10}$

Self-immolative spacers commonly reported in the literature are mainly based on phenyl cores, as aniline and phenol derivatives. The nitrogen or oxygen atom, typically involved in a bond with various protecting groups, triggers the self-immolation process. Here we used a phenol core, acting as a nucleophile during the elimination step. Two representative series seemed particularly interesting for cyclisation studies (Scheme 1):

Trimethyl-lock derivatives ${ }^{13-18}$ via 6-exo-trig-cyclisation. These are the most widely used cyclisation-based spacers.

2-Hydroxyphenyl carbamate (2-HPC) derivatives ${ }^{19}$ via 5-exotrig cyclisation. These derivatives are less popular but are good models to study the influence of substituents on the selfimmolation rate (4 substituents were selected: methoxy, nitro, $\mathrm{H}$, bromide).

In order to analyse the stoichiometry and kinetics of cyclisation reactions, we used 1,3-dichloro-9,9-dimethyl-9H-acridin-2 (7)-one (DDAO in Scheme 1) as a fluorescent reporter. This moiety does not emit fluorescence in caged precursors, but emits strongly in the red-wavelength region in the free phenol state.

\section{Trimethyl-lock synthesis}

The synthesis of the trimethyl-lock derivative (see Scheme 2) began by an esterification and an aromatic electrophilic substitution of 3,5-dimethylphenol with 3,3-dimethylacrylic acid under heating and acidic conditions to form the corresponding dihydrocoumarin. The lactone was then reduced by lithium aluminium hydride, leading to the free alcohol. These two steps are common in trimethyl lock derivative syntheses. The following steps have been adapted to produce our own compound. The phenol was engaged in an etherification reaction with 6-nitroveratryl bromide, and then the primary alcohol was successively oxidized to aldehyde and carboxylic acid by, respectively, pyridinium dichromate and a Pinnick oxidation. The acid was finally converted to acyl chloride by phosgene and esterified by DDAO to give the desired ester with an overall yield of $72 \%$.
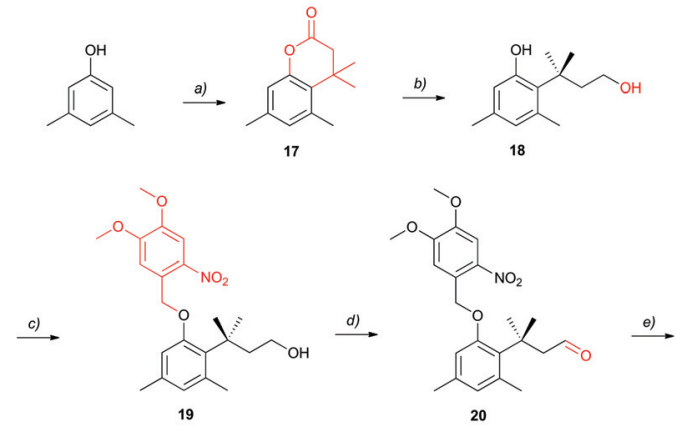

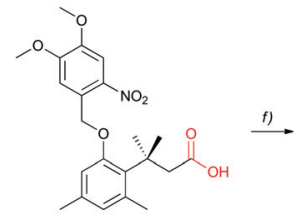

21

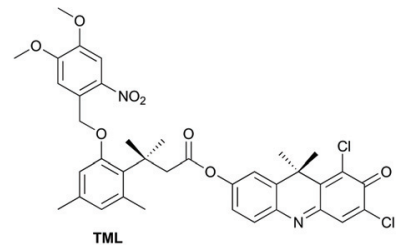

Scheme 2 Synthesis of the trimethyl-lock derivative. Reagents and conditions: (a) 3.3-dimethylacrylic acid, methane sulfonic acid, toluene, $85^{\circ} \mathrm{C}$ (99\%); (b) $\mathrm{LiAlH}_{4}, \mathrm{THF}, 0{ }^{\circ} \mathrm{C}$ to rt (93\%); (c) 4,5-dimethoxy-2-nitrobenzyl bromide, $\mathrm{Cs}_{2} \mathrm{CO}_{3}, \mathrm{THF}$, rt (93\%); (d) pyridinium dichromate (PDC), $\mathrm{CH}_{2} \mathrm{Cl}_{2}$, rt (95\%); (e) $\mathrm{NaClO}_{2}, \mathrm{NaH}_{2} \mathrm{PO}_{4}$, 2-methyl-2-butene, acetone/ tBuOH/water, rt (89\%); (f) 1. phosgene, THF, rt; 2. NEt 3 , 4-dimethylaminopyridine (DMAP), DDAO, THF, rt (quant.).

\section{Phenol-carbamate syntheses}

The general synthesis of the 2-HPC (see Scheme 3) derivatives started by the aniline protection of the corresponding commercial 2-aminophenol with a Boc group. The selective protection of the aniline function versus phenol came from a metha-

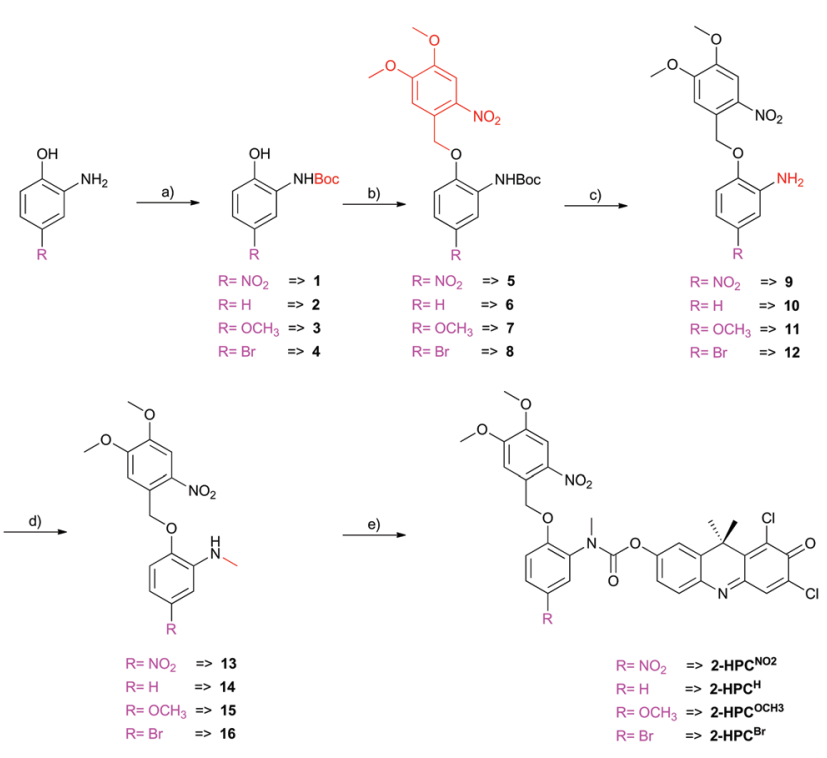

Scheme 3 General synthesis of 2-HPC derivatives, with $\mathrm{R}$ as a para substituent $\left(\mathrm{H}, \mathrm{Br}, \mathrm{NO}_{2}, \mathrm{OCH}_{3}\right)$. Reagents and conditions: (a) $\mathrm{Boc}_{2} \mathrm{O}$, $\mathrm{K}_{2} \mathrm{CO}_{3}, \mathrm{THF}, \mathrm{H}_{2} \mathrm{O}$, rt (96\% to quant.); (b) 4,5-dimethoxy-2-nitrobenzyl bromide, $\mathrm{Cs}_{2} \mathrm{CO}_{3}$, THF, rt (71\% to quant.); (c) trifluoroacetic acid (TFA), $\mathrm{CH}_{2} \mathrm{Cl}_{2}$, rt (quant.); (d) $\mathrm{CH}_{3} \mathrm{I}_{2} \mathrm{~K}_{2} \mathrm{CO}_{3}, \mathrm{DMF}$, rt (42 to $50 \%$ ); (e) 1. phosgene, THF, rt; 2. NEt 3 , DMAP, DDAO, THF, rt (quant.). 
nolysis step that led to carbonate cleavage keeping the carbamate intact. Etherification of the phenol position with 6-nitroveratryl bromide was carried out under basic conditions, and then the Boc protecting group was removed under acidic conditions using a $1: 1$ TFA: $\mathrm{CH}_{2} \mathrm{Cl}_{2}$ mixture. This liberated the free aniline which was then monomethylated by iodomethane. Other methods of selective monomethylation (such as reductive amination or use of a soft methylating agent like dimethyl carbonate) showed incompatibilities with our compounds. Finally, the secondary aniline was treated with phosgene, and the resulting carbamoyl chloride reacted with the phenol function of the DDAO to form the desired carbamate (overall yield, from the commercial anilines: 32 to $47 \%$, depending on the substituents). These carbamates consisted of two rotamers (N-C bond).

\section{Cyclisation rates}

For the kinetic measurements, our working hypothesis was a two-step process as depicted in Scheme 4. The first step is a photocleavage of the caged precursor, associated with $k_{1}$, the rate constant of uncaging, and the intramolecular cyclisation, where $k_{2}$ is the rate constant for the self-immolation step.

We followed the same procedure as previously described. ${ }^{11}$ The principle is to irradiate the compound at $365 \mathrm{~nm}$ and to follow the liberation of the released DDAO by fluorimetry in a quartz cuvette under temperature $(293 \mathrm{~K}$ ) and $\mathrm{pH}$ control. The experimental data were then fitted with the kinetic model to retrieve the values of $k_{1}$ and $k_{2}$. The results are reported in Table 1.

\section{Discussion}

Results showed that cyclisation generally occurs in the minute range, for both trimethyl lock and 2-HPC derivatives; disassembly times were close to each other, showing only a factor of six between the largest and the smallest. For the nitro

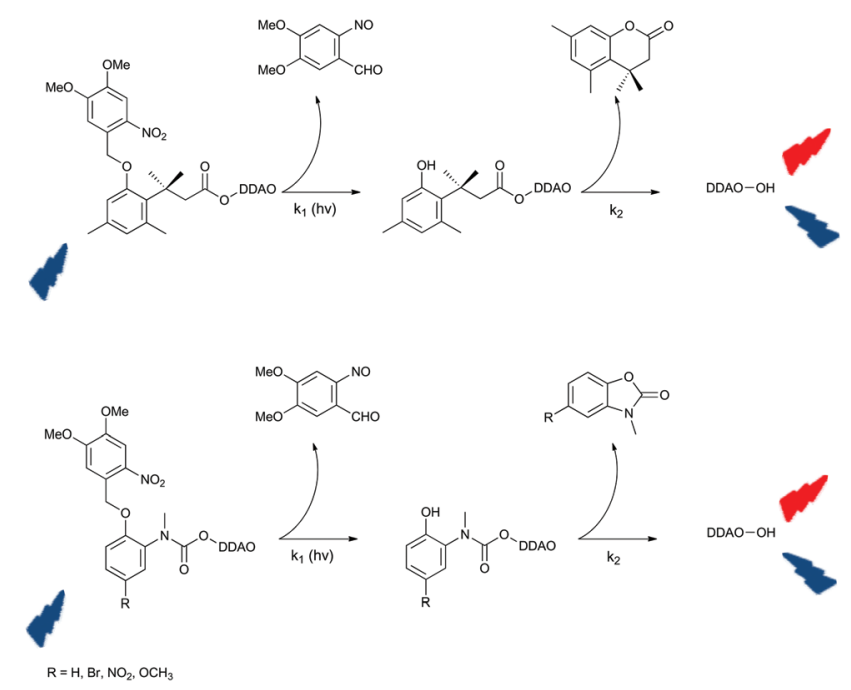

Scheme 4 Kinetic models for disassembly from photoactivation to self-immolation of trimethyl-lock (top) and 2-HPC (bottom) derivatives.
Table 1 Kinetic constants of trimethyl lock and 2-HPC derivatives. See the ESI for equations and methods; where $\tau=1 / k_{2}, k_{2}$ rate constant of the self-immolation step. $\mathrm{p} K_{\mathrm{a}}$ determination was carried out by following absorbance of protected aniline 1, 2, 3, 4 as a function of $\mathrm{pH}$, applying the same method as in Alouane et al. ${ }^{10,11}$

\begin{tabular}{lclc}
\hline Compound & $\mathrm{p} K_{\mathrm{a}}$ of the phenol & $k_{2}\left(\mathrm{~min}^{-1}\right)$ & $\tau_{2}(\mathrm{~min})$ \\
\hline 2-HPC $^{\mathrm{NO2}}$ (pH 5) & $7.6 \pm 0.1$ & $(5.2 \pm 0.5) 10^{-2}$ & 19 \\
2-HPC $^{\mathrm{NO} 2}$ (pH 8) & $7.6 \pm 0.1$ & $(6.6 \pm 0.5) 10^{-2}$ & 15 \\
2-HPC $^{\mathrm{Br}}$ (pH 8) & $10.0 \pm 0.1$ & $(1.1 \pm 0.3) 10^{-1}$ & 9 \\
2-HPC $^{\mathbf{H}}$ (pH 8) & $10.7 \pm 0.1$ & $(1.9 \pm 0.2) 10^{-1}$ & 5 \\
2-HPC $^{\text {OCH3 }}$ (pH 8) & $10.8 \pm 0.1$ & $(2.9 \pm 0.3) 10^{-1}$ & 3.5 \\
TML (pH 8) $^{\text {pH }}$ & $10.2 \pm 0.1$ & $(3.3 \pm 0.3) 10^{-1}$ & 3
\end{tabular}

derivative of 2-HPC, which is the only derivative with a physiological $\mathrm{p} K_{\mathrm{a}}$ (7.6), we did not observe any significant dependence on $\mathrm{pH}$ : kinetics at $\mathrm{pH} 5$ and $\mathrm{pH} 8$ are close, showing only a $20 \%$ difference.

The trimethyl lock was important because of the large number of publications using it. The popularity of the trimethyl-lock is due to the Thorpe-Ingold effect, which is supposed to allow rapid intramolecular cyclisation. This steric effect is induced by the unfavourable interaction between the methyl at the meta position on the phenol core and the two geminal methyls on the alkyl chain ( $\beta$ position from the ester). Because of this steric hindrance, a conformation was favoured, making the carbonyl of the ester and the phenol closer. This vicinity was already known to drastically increase the cyclisation rate ${ }^{20}$ but the kinetics of the process was still unknown. We measured only the rate constant of the TML, in line with the huge interest to this linkage compared to other carbonyl species (amide for instance). ${ }^{20}$ The measured lactonisation time was around $3 \mathrm{~min}$ for this ester derivative.

The 2-HPC series was chosen to study the cyclisation rates of carbamates, which are relatively resistant to enzymes such as esterases or peptidases. The spacers have been modulated by variation of the substituents on the phenol core. The results enabled us to determine the effects of the substituent and of the protonation state on the kinetics of cyclisation. By lowering the $\mathrm{p} K_{\mathrm{a}}$ via a withdrawing group, the proportion of phenolate is increased; the phenolate anion is more nucleophilic than the phenol and so able to accelerate the cyclisation process. We focused our attention on the nitro derivative, which exhibits a $\mathrm{p} K_{\mathrm{a}}$ close to the physiological $\mathrm{pH}$; in order to be in vivo/ in vitro deprotonated. We observed that the kinetics of the selfimmolation did not change significantly by deprotonating the nitrophenol, which led us to conclude that the gain of nucleophilicity was partially compensated by the conjugation of the phenolate with the nitro group. More generally, the more electron-donors the cycle has at the para position, the faster the cyclisation occurs in line with an enhanced nucleophilicity of the phenol: compared to the $\mathrm{H}$ derivative (para-hydrogen), we observed a relative acceleration with electron-donor groups and a moderate slow-down with electron-withdrawing groups. According to the literature, in both aliphatic ${ }^{21}$ and aromatic ${ }^{22}$ derivatives, nucleophilicity enhancement is a critical factor for 

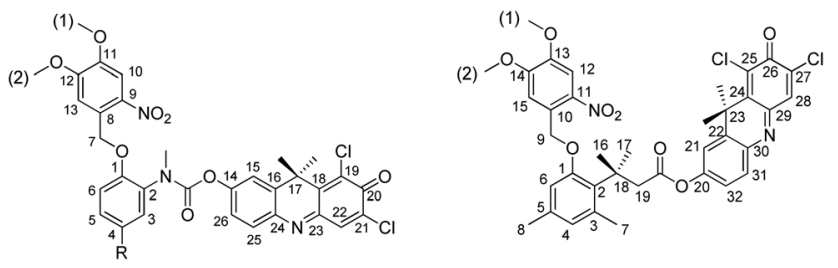

Scheme 5 Numeration for $\mathrm{C}^{13}$ and $\mathrm{H}^{1}$ signals.

the cyclisation rate. Indeed, in every series (including ours, phenol-based ones), electron-donating groups made the nucleophile (oxygen or nitrogen in previously cited works) more reactive. Adding this to other parameters, like decreasing $\mathrm{p} K_{\mathrm{a}}$ of the leaving group, changing the heteroatom, promoting cyclisation at high $\mathrm{pH}$, choosing a better electrophilic carbonyl, or increasing temperature, various cyclisation-based spacer can be generated with half-times close to the minute range, or even less.

Eventually, this work and our previous work ${ }^{11,12}$ show that self-immolation in elimination-based spacers is much more rapid and substituent sensitive than in cyclisation-based spacers.

\section{Conclusions}

We investigated the self-immolation kinetics of common cyclisation-based self-immolative spacers. They exhibited releasetimes within the range of 1-10 minute at room temperature. Steric effects were more stringent than electronic effects to modulate the self-immolation rate. The comparison between cyclisation and elimination-driven self-immolation rates (as reported by Alouane et al. $)^{11,12}$ further suggested that the latter mechanism has more potential for modulating kinetics.

\section{Experimental}

The commercially available chemicals were used without further purification. Anhydrous solvents were freshly distilled before use. Low actinic glassware and an aluminium film were used for all experiments involving compounds bearing the nitroveratryl moiety. Column chromatography (CC): silica gel 60 (0.040-0.063 mm) Merck. Analytical and thin layer chromatography (TLC): Merck silica gel 60 F-254 precoated plates; detection by UV ( 254 and $365 \mathrm{~nm}$ ). ${ }^{1} \mathrm{H}$ NMR spectra were recorded at $300 \mathrm{MHz} .{ }^{13} \mathrm{C}$ NMR spectra were recorded at $75 \mathrm{MHz}$ with complete proton decoupling; chemical shifts $(\delta)$ in ppm related to a protonated solvent as the internal reference $\left({ }^{1} \mathrm{H}: \mathrm{CHCl}_{3}\right.$ in $\mathrm{CDCl}_{3}, 7.26 \mathrm{ppm} ;{ }^{13} \mathrm{C}:{ }^{13} \mathrm{CDCl}_{3}$ in $\mathrm{CDCl}_{3}, 77.0 \mathrm{ppm}$; coupling constants $J$ in $\mathrm{Hz}$; current notations are used for multiplicity (s: singlet; bs: broad singlet; d: doublet; dd: double doublet; t: triplet; q: quadruplet; m: multiplet)) (Scheme 5).

\section{General procedure}

Aniline protection $(1,2,3,4)$. To a solution of 2-aminophenol derivative (1 eq.) in THF/water (1:1 proportion) was added $\mathrm{K}_{2} \mathrm{CO}_{3}$ (5 eq.) and di-tert-butyl dicarbonate (2.6 eq.). After stirring for $4 \mathrm{~h}$ at room temperature, the mixture was neutralized (acetic acid 100\%, until pH 7), the organic layer was diluted with EtOAc and washed with water and brine, then dried on $\mathrm{MgSO}_{4}$, filtered and concentrated under reduced pressure. The residue was resolubilized in $\mathrm{MeOH}$ and we added $\mathrm{K}_{2} \mathrm{CO}_{3}$ (5 eq.) and stirred at room temperature until no more evolution is observed (as seen on TLC, between $1 \mathrm{~h}$ $30 \mathrm{~min}$ and $4 \mathrm{~h}$ ). The same work-up as previously applied to obtain the crude product.

Nitroveratryl coupling $(5,6,7,8)$. 4,5-Dimethoxy-2-nitrobenzyl bromide (1 eq.) was introduced into a solution containing the carbamate (1 eq.), and $\mathrm{Cs}_{2} \mathrm{CO}_{3}$ (1.5 eq.) in THF. The mixture was stirred overnight at room temperature, and then neutralized to $\mathrm{pH} 7$ by $1 \mathrm{M} \mathrm{HCl}$, washed with water and then with brine, dried on $\mathrm{MgSO}_{4}$, filtered and evaporated. After purification on silica gel column chromatography, the corresponding ether was obtained.

Aniline deprotection (9, 10, 11, 12). The protected aniline (1 eq.) was solubilized in dichloromethane/trifluoroacetic acid ( $1: 1$ volume ratio) and stirred at room temperature for $30 \mathrm{~min}$. After evaporation, the residue was taken up in EtOAc and washed with aqueous $\mathrm{K}_{2} \mathrm{CO}_{3}$ solution, water, and brine, then dried on $\mathrm{MgSO}_{4}$, filtered and the solvent was removed in vacuo. The unprotected secondary anilines were isolated without further purification.

Aniline methylation $(\mathbf{1 3}, \mathbf{1 4}, \mathbf{1 5}, \mathbf{1 6})$. The secondary anilines (1 eq.), $\mathrm{K}_{2} \mathrm{CO}_{3}$ (1.5 eq.), and iodomethane (1 eq.) in DMF were stirred at room temperature under argon until reaction showed no more evolution on TLC. The solvent was removed and the residue was solubilized in EtOAc. The organic layer was washed with water several times, and then with brine; and finally dried on $\mathrm{MgSO}_{4}$, filtered and concentrated to dryness. The secondary anilines were obtained after purification by column chromatography on silica gel.

Coupling with DDAO (2-HPC). To a solution of $N$-methylaniline (1 eq.) in anhydrous THF, was carefully injected an excess of phosgene $30 \%$ in toluene $(500 \mu \mathrm{L})$ under argon. The mixture was stirred at room temperature for $20 \mathrm{~min}$. The remaining phosgene was eliminated by an argon flux (warning, highly toxic), and then the solution was added to a solution of 1,3-dichloro-7-hydroxy-9,9-dimethylacridin-2 (9H)-one (0.055 mmol), 4-dimethylaminopyridine $(0.055 \mathrm{mmol})$ and an excess of triethylamine $(1 \mathrm{~mL})$, in THF. The resulting solution was stirred under argon at room temperature overnight. The solvent was evaporated, and then the residue was taken up in dichloromethane; the organic phase was washed with a solution of $\mathrm{K}_{2} \mathrm{CO}_{3}$, water and brine; dried on $\mathrm{MgSO}_{4}$, filtered and concentrated in vacuo. The final carbamates were purified by HPLC using 80 to $90 \%$ gradient of $\mathrm{CH}_{3} \mathrm{CN} /$ water/0.1\% TFA, Waters XBridge ${ }^{\circledR}$ Prep C18 $5 \mu \mathrm{m}$ $\mathrm{OBD}^{\mathrm{TM}} 30 \times 150 \mathrm{~mm}$ Column.

tert-Butyl(2-hydroxy-5-nitrophenyl)carbamate (1). The carbamate 1 was obtained without purification as a dark yellow powder (330 mg, 99\%); rf 0.57 (40\% EtOAc/cyclohexane); mp $111{ }^{\circ} \mathrm{C}$; IR $\left(\mathrm{cm}^{-1}\right) 3264,1765,1530,1151 ; \delta_{\mathrm{H}} 1.54(\mathrm{~s}, 9 \mathrm{H}, t \mathrm{Bu})$, 
3.81 (bs, 1H, OH), 7.9 (dd, $1 \mathrm{H}, J=2.67 / 8.91 \mathrm{~Hz}, \mathrm{H}_{6}$ ), 8.06 (d, $\left.1 \mathrm{H}, J=2.64, \mathrm{H}_{3}\right), 8.11\left(\mathrm{dd}, 1 \mathrm{H}, 2.70 / 8.94 \mathrm{~Hz}, \mathrm{H}_{5}\right), 8.23(\mathrm{bs}, 1 \mathrm{H}$, $\mathrm{NH}) ; \delta_{\mathrm{C}} 28.2(t \mathrm{Bu}), 85.2\left(\mathrm{Cq}\right.$ Boc), $117.2\left(\mathrm{C}_{3}\right), 121.1\left(\mathrm{C}_{6}\right), 126.2$ $\left(\mathrm{C}_{5}\right), 126.8\left(\mathrm{C}_{2}\right), 151.8\left(\mathrm{C}_{4}\right), 153.4(\mathrm{C}=\mathrm{O}), 158.6\left(\mathrm{C}_{1}\right) ; \mathrm{m} / \mathrm{z}$ 155, 199, $377[\mathrm{M}+\mathrm{Na}]^{+}$. HRMS, calculated: $m / z$ 277.0793, found: $m / z 277.0792\left([\mathrm{M}+\mathrm{Na}]^{+}\right), 278.0825\left(\left[\mathrm{MN}^{15}+\mathrm{Na}\right]^{+}\right)$.

tert-Butyl(2-((4,5-dimethoxy-2-nitrobenzyl)oxy)-5-nitrophenyl) carbamate (5). The crude product was purified by column chromatography on silica gel (20\% EtOAc/cyclohexane) to give the ether 5 as a dark red solid (548 mg, 94\%) rf $0.23(20 \%$ EtOAc/cyclohexane); mp $122{ }^{\circ} \mathrm{C}$; IR $\left(\mathrm{cm}^{-1}\right)$ 2979, 1785, 1519; $\delta_{\mathrm{H}} 1.36(9 \mathrm{H}, \mathrm{s}, t \mathrm{Bu}), 3.97\left(3 \mathrm{H}, \mathrm{s}, \mathrm{OCH}_{3}(1)\right), 4.03\left(3 \mathrm{H}, \mathrm{s}, \mathrm{OCH}_{3}\right.$ (2)), $5.65\left(1 \mathrm{H}, \mathrm{s}, \mathrm{H}_{7}\right), 7.15\left(1 \mathrm{H}, \mathrm{d}, 9.12 \mathrm{~Hz}, \mathrm{H}_{6}\right), 7.36(1 \mathrm{H}, \mathrm{s}$, $\left.\mathrm{H}_{13}\right), 7.81\left(1 \mathrm{H}, \mathrm{s}, \mathrm{H}_{10}\right), 8.13\left(1 \mathrm{H}, \mathrm{d}, 2.55 \mathrm{~Hz}, \mathrm{H}_{3}\right), 8.26(1 \mathrm{H}$, dd, $\left.2.58 / 9.06 \mathrm{~Hz}, \mathrm{H}_{5}\right) ; \delta_{\mathrm{C}} 27.8\left(\mathrm{CH}_{3} \mathrm{Boc}\right), 56.4-57.2\left(\mathrm{OCH}_{3}(1) \&\right.$ (2)), $67.8\left(\mathrm{C}_{7}\right), 83.4$ (Cq Boc), $108\left(\mathrm{C}_{3}\right), 108.5\left(\mathrm{C}_{13}\right), 111.9\left(\mathrm{C}_{5}\right)$, $125.4\left(\mathrm{C}_{6}\right), 125.5\left(\mathrm{C}_{2}\right), 127.4\left(\mathrm{C}_{8}\right), 129.2\left(\mathrm{C}_{10}\right), 138.3\left(\mathrm{C}_{9}\right), 141.5$ $\left(\mathrm{C}_{11}\right), 148.1\left(\mathrm{C}_{4}\right), 150.8(\mathrm{C}=\mathrm{O}$ Boc$), 154.7\left(\mathrm{C}_{12}\right), 158\left(\mathrm{C}_{1}\right) ; \mathrm{m} / \mathrm{z}$ 196, $472[\mathrm{M}+\mathrm{Na}]^{+}$. HRMS, calculated: $m / z$ 472.1334, found: $m / z 472.1326\left([\mathrm{M}+\mathrm{Na}]^{+}\right)$.

2-((4,5-Dimethoxy-2-nitrobenzyl)oxy)-5-nitroaniline (9). The aniline 9 was directly obtained pure as a deeply red solid (427 mg, quantitative yield) rf 0.18 (30\% EtOAc/cyclohexane); mp $187{ }^{\circ} \mathrm{C}$; IR $\left(\mathrm{cm}^{-1}\right)$ 3368, 2922, 2851, 1783, 1514; $\delta_{\mathrm{H}} 3.92$ $\left(3 \mathrm{H}, \mathrm{s}, \mathrm{OCH}_{3}(1)\right), 3.97\left(3 \mathrm{H}, \mathrm{s}, \mathrm{OCH}_{3}(2)\right), 5.62\left(1 \mathrm{H}, \mathrm{s}, \mathrm{H}_{7}\right), 7.15$ $\left(1 \mathrm{H}, \mathrm{d}, 9.12 \mathrm{~Hz}, \mathrm{H}_{6}\right), 7.36\left(1 \mathrm{H}, \mathrm{s}, \mathrm{H}_{13}\right), 7.81\left(1 \mathrm{H}, \mathrm{s}, \mathrm{H}_{10}\right), 8.13$ $\left(1 \mathrm{H}, \mathrm{d}, 2.55 \mathrm{~Hz}, \mathrm{H}_{3}\right), 8.26\left(1 \mathrm{H}, \mathrm{dd}, 2.58 / 9.06 \mathrm{~Hz}, \mathrm{H}_{5}\right) ; \delta_{\mathrm{C}} 56.6$ $\left(\mathrm{OCH}_{3}(1) \&(2)\right), 68.1\left(\mathrm{C}_{7}\right), 108.2\left(\mathrm{C}_{3}\right), 109.5\left(\mathrm{C}_{5} \& \mathrm{C}_{13}\right), 111.1$ $\left(\mathrm{C}_{6}\right), 114.8\left(\mathrm{C}_{8}\right), 127.4\left(\mathrm{C}_{10}\right), 136.9\left(\mathrm{C}_{2}\right), 139.5\left(\mathrm{C}_{4}\right), 142.5\left(\mathrm{C}_{9}\right)$, $148.3\left(\mathrm{C}_{11}\right), 150.1\left(\mathrm{C}_{1}\right), 154\left(\mathrm{C}_{12}\right) ; \mathrm{m} / z$ 196, 350, $545[\mathrm{M}+\mathrm{H}]^{+}$. HRMS, calculated: $\mathrm{m} / \mathrm{z}$ 350.0910, found: $\mathrm{m} / \mathrm{z} 350.0982$ $\left([\mathrm{M}+\mathrm{H}]^{+}\right)$.

2-((4,5-Dimethoxy-2-nitrobenzyl)oxy)-N-methyl-5-nitroaniline (13). The aniline $\mathbf{1 3}$ was obtained after purification by column chromatography on silica gel (15\% EtOAc/cyclohexane) as a yellow solid (170 mg, 40\%); rf 0.23 (20\% EtOAc/cyclohexane); mp $197{ }^{\circ} \mathrm{C}$; IR $\left(\mathrm{cm}^{-1}\right) 3424,2921,2851,1271,1219 ; \delta_{\mathrm{H}} 2.96(\mathrm{~s}$, $3 \mathrm{H}, \mathrm{NCH}_{3}$ ), $3.90\left(\mathrm{~s}, 3 \mathrm{H}, \mathrm{OCH}_{3}(1)\right), 3.98$ (s, 3H, $\left.\mathrm{OCH}_{3}(2)\right), 5.62$ $\left(\mathrm{s}, 2 \mathrm{H}, \mathrm{H}_{7}\right), 6.78$ (d, $\left.1 \mathrm{H}, J=9 \mathrm{~Hz}, \mathrm{H}_{6}\right), 7.03\left(\mathrm{~s}, 1 \mathrm{H}, \mathrm{H}_{13}\right), 7.43$ (d, $1 \mathrm{H}, J=3 \mathrm{~Hz}, \mathrm{H}_{3}$ ), 7.58 (dd, $\left.1 \mathrm{H}, J=3 / 9 \mathrm{~Hz}, \mathrm{H}_{5}\right), 7.77(\mathrm{~s}, 1 \mathrm{H}$, $\left.\mathrm{H}_{10}\right) ; \delta_{\mathrm{C}} 30.2\left(\mathrm{NCH}_{3}\right), 56.5\left(\mathrm{OCH}_{3}(1) \&(2)\right), 68.2\left(\mathrm{C}_{7}\right), 103.9$ $\left(\mathrm{C}_{3}\right), 108.3\left(\mathrm{C}_{5}\right), 109.7\left(\mathrm{C}_{13}\right), 109.9\left(\mathrm{C}_{6} / \mathrm{C}_{8}\right), 113\left(\mathrm{C}_{10}\right), 127.2\left(\mathrm{C}_{2}\right)$, $139.7\left(\mathrm{C}_{9}\right), 143.1\left(\mathrm{C}_{11}\right), 148.4\left(\mathrm{C}_{12}\right), 148.9\left(\mathrm{C}_{1}\right), 153.9\left(\mathrm{C}_{4}\right) ; \mathrm{m} / \mathrm{z}$ 196, $364[\mathrm{M}+\mathrm{H}]^{+}$. HRMS, calculated: $m / z$ 364.1100, found: $m / z$ $364.1141\left([\mathrm{M}+\mathrm{H}]^{+}\right)$.

6,8-Dichloro-9,9-dimethyl-7-oxo-7,9-dihydroacridin-2-yl(2-)(4,5dimethoxy-2-nitrobenzyl)oxy)-5-nitrophenyl)(methyl)carbamate $\left(2-H_{P C} C^{N 2}\right)$. The crude product was purified by column chromatography on silica gel (100\% dichloromethane) to give the final carbamate $\mathbf{2}-\mathbf{H P C}^{\mathbf{N O 2}}$ (38.4 $\mathrm{mg}$, quantitative yield) as a yellow solid; rf 0.14 (20\% EtOAc/cyclohexane); mp $216{ }^{\circ} \mathrm{C}$; IR $\left(\mathrm{cm}^{-1}\right)$ 2922, 2851, 1787, 1656, 1254, 1220; $\delta_{\mathrm{H}} 1.67(\mathrm{~s}, 6 \mathrm{H}$, $\mathrm{CH}_{3}$ DDAO), $3.42\left(\mathrm{~s}, 3 \mathrm{H}, \mathrm{NCH}_{3}\right.$ ), 3.90 (s, 3H, $\left.\mathrm{OCH}_{3}(1)\right), 4$ (s, $\left.3 \mathrm{H}, \mathrm{OCH}_{3}(2)\right), 5.71\left(\mathrm{~s}, 2 \mathrm{H}, \mathrm{H}_{7}\right), 6.88\left(\mathrm{~d}, 1 \mathrm{H}, J=8.1 \mathrm{~Hz}, \mathrm{H}_{6}\right), 6.9$ $\left(\mathrm{s}, 1 \mathrm{H}, \mathrm{H}_{13}\right), 7.31\left(\mathrm{~s}, 1 \mathrm{H}, \mathrm{H}_{22}\right), 7.35\left(\mathrm{~d}, 1 \mathrm{H}, J=4.32 \mathrm{~Hz}, \mathrm{H}_{5}\right), 7.5$ (s, $1 \mathrm{H}, \mathrm{H}_{25}$ ), 7.84 (d, $\left.1 \mathrm{H}, J=4.32 \mathrm{~Hz}, \mathrm{H}_{3}\right), 8.34\left(\mathrm{~s}, 2 \mathrm{H}, \mathrm{H}_{15} / \mathrm{H}_{26}\right)$, $8.37\left(\mathrm{~s}, 1 \mathrm{H}, \mathrm{H}_{10}\right) ; \delta_{\mathrm{C}} 26.5\left(\mathrm{CH}_{3} \mathrm{DDAO}\right), 30.9\left(\mathrm{NCH}_{3}\right), 56.5 / 56.9$ $\left(\mathrm{OCH}_{3} 1\right.$ and 2$), 68.3\left(\mathrm{C}_{3}\right), 108.2\left(\mathrm{C}_{5}\right), 108.3\left(\mathrm{C}_{3}\right), 108.4\left(\mathrm{C}_{13}\right)$, $112.8\left(\mathrm{C}_{15}\right), 119.7\left(\mathrm{C}_{6}\right), 121.2\left(\mathrm{C}_{25}\right), 124.8\left(\mathrm{C}_{26}\right), 125.7\left(\mathrm{C}_{2}\right), 131.8$ $\left(\mathrm{C}_{8}\right), 133\left(\mathrm{C}_{10}\right), 135.6\left(\mathrm{C}_{19} / \mathrm{C}_{21}\right), 138.4\left(\mathrm{C}_{9}\right), 136.8\left(\mathrm{C}_{4} / \mathrm{C}_{24}\right), 138.4$ $\left(\mathrm{C}_{16}\right), 139.4\left(\mathrm{C}_{11} / \mathrm{C}_{12}\right), 148.5\left(\mathrm{C}_{18}\right), 149.8\left(\mathrm{C}_{14}\right), 153.5\left(\mathrm{C}_{22}\right), 153.6$ $\left(\mathrm{C}_{23}\right), 158.5\left(\mathrm{C}_{1}\right), 173.1\left(\mathrm{C}_{20}\right), 207(\mathrm{C}=\mathrm{O}$ carbamate $) ; \mathrm{m} / \mathrm{z} 196$, 289, 371, 447, $697[\mathrm{M}+\mathrm{H}]^{+}$. HRMS, calculated: $m / z$ 697.1096, found: $m / z 697.1105\left([\mathrm{M}+\mathrm{H}]^{+}\right), 699.1088\left(\left[\mathrm{MCl}^{37}+\mathrm{H}\right]^{+}\right)$.

tert-Butyl(2-hydroxyphenyl)carbamate (2). The carbamate 2 was obtained as a dark yellow powder (919 mg, 96\%); rf 0.7 (40\% EtOAc/cyclohexane); mp $142{ }^{\circ} \mathrm{C}$; IR $\left(\mathrm{cm}^{-1}\right)$ 3284, 1690, 1147; $\delta_{\mathrm{H}} 1.53(\mathrm{~s}, 9 \mathrm{H}, t \mathrm{Bu}), 6.77$ (bs, 1H, OH), $6.85(\mathrm{~d}, 1 \mathrm{H}, J=$ $14.7 \mathrm{~Hz}, \mathrm{H}_{6}$ ), 6.94 (d, 1H, $14.6 \mathrm{~Hz}, \mathrm{H}_{4}$ ), 6.98 (d, 1H, $9.2 \mathrm{~Hz}, \mathrm{H}_{5}$ ), 7.15 (d, $1 \mathrm{H}, 7.5 \mathrm{~Hz}, \mathrm{H}_{3}$ ); $\delta_{\mathrm{C}} 28.3\left(\mathrm{CH}_{3} \mathrm{Boc}\right.$ ), 82.1 (Cq Boc), 118.9 $\left(\mathrm{C}_{6}\right), 120.8\left(\mathrm{C}_{4}\right), 121.4\left(\mathrm{C}_{2}\right), 125.7\left(\mathrm{C}_{3 / 5}\right), 147.5\left(\mathrm{C}_{1}\right), 155.1(\mathrm{C}=\mathrm{O}$ Boc); $m / z$ 176, $232[\mathrm{M}+\mathrm{Na}]^{+}$. HRMS, calculated: $m / z$ 232.0917, found: $m / z 232.0943\left([\mathrm{M}+\mathrm{Na}]^{+}\right)$.

tert-Butyl(2-((4,5-dimethoxy-2-nitrobenzyl)oxy)phenyl)carbamate (6). The crude product was purified by column chromatography on silica gel (20\% EtOAc/cyclohexane) to give the ether 6 as a light yellow solid (376.1 mg, quantitative yield); rf 0.3 (20\% EtOAc/cyclohexane); mp $137{ }^{\circ} \mathrm{C}$; IR $\left(\mathrm{cm}^{-1}\right)$ 3452, 2943, 2897, 1730, 1221, 1150; $\delta_{\mathrm{H}} 1.52(\mathrm{~s}, 9 \mathrm{H}, t \mathrm{Bu}), 3.92\left(\mathrm{~s}, 3 \mathrm{H}, \mathrm{OCH}_{3}\right.$ (1)), 3.96 (s, 3H, $\left.\mathrm{OCH}_{3}(2)\right), 5.54\left(\mathrm{~s}, 2 \mathrm{H}, \mathrm{H}_{7}\right), 6.84$ (dd, $1 \mathrm{H}, J=$ 1.26/14.7 Hz, $\mathrm{H}_{6}$ ), 6.9 (dd, $1 \mathrm{H}, J=1.3 / 14.1 \mathrm{~Hz}, \mathrm{H}_{4}$ ), 6.95 (dd, $1 \mathrm{H}, J=1.3 / 14 \mathrm{~Hz}, \mathrm{H}_{5}$ ), 7.12 (bs, $\left.1 \mathrm{H}, \mathrm{NH}\right), 7.18$ (s, 1H, $\left.\mathrm{H}_{13}\right), 7.77$ $\left(\mathrm{s}, 1 \mathrm{H}, \mathrm{H}_{10}\right), 8.07\left(\mathrm{~d}, 1 \mathrm{H}, J=7 \mathrm{~Hz}, \mathrm{H}_{3}\right) ; \delta_{\mathrm{C}} 28.5\left(\mathrm{CH}_{3} \mathrm{Boc}\right), 55.9$ $\left(\mathrm{OCH}_{3}\right.$ (1) \& (2)), $67.7\left(\mathrm{C}_{7}\right), 82.3$ (Cq Boc), $109.5\left(\mathrm{C}_{13}\right), 118.7$ $\left(\mathrm{C}_{8}\right), 119.1\left(\mathrm{C}_{6}\right), 121\left(\mathrm{C}_{4}\right), 121.6\left(\mathrm{C}_{2}\right), 122.2\left(\mathrm{C}_{10}\right), 125.9\left(\mathrm{C}_{3 / 5}\right)$, $136.5\left(\mathrm{C}_{9}\right), 139.1\left(\mathrm{C}_{11}\right), 145.7\left(\mathrm{C}_{12}\right), 147.7\left(\mathrm{C}_{1}\right), 155.3(\mathrm{C}=\mathrm{O}$ Boc); $m / z$ 196, 305, 349, $405[\mathrm{M}+\mathrm{H}]^{+}, 427\left([\mathrm{M}+\mathrm{Na}]^{+}\right)$. HRMS, calculated: $m / z$ 405.1617, found: $m / z 405.1661\left([\mathrm{M}+\mathrm{H}]^{+}\right)$.

2-((4,5-Dimethoxy-2-nitrobenzyl)oxy)aniline (10). The aniline 10 was obtained as a yellow solid $(137.7 \mathrm{mg}$, quantitative yield); rf 0.24 (30\% EtOAc/cyclohexane); mp $115{ }^{\circ} \mathrm{C}$; IR $\left(\mathrm{cm}^{-1}\right)$ 3453, 2946, 2907, 1221, 1151; $\delta_{\mathrm{H}} 3.90$ (s, 3H, $\left.\mathrm{OCH}_{3}(1)\right), 3.93$ (s, $\left.3 \mathrm{H}, \mathrm{OCH}_{3}(2)\right), 5.49$ (s, 2H, H ), 6.64 (dd, $1 \mathrm{H}, J=1.3 / 14.7 \mathrm{~Hz}$, $\left.\mathrm{H}_{6}\right), 6.69\left(\mathrm{dd}, 1 \mathrm{H}, J=1.28 / 13.7 \mathrm{~Hz}, \mathrm{H}_{4}\right), 6.76(\mathrm{dd}, 1 \mathrm{H}, J=1.3 / 14$ $\left.\mathrm{Hz}, \mathrm{H}_{5}\right), 6.8\left(\mathrm{dd}, 1 \mathrm{H}, J=1.7 / 14.2 \mathrm{~Hz}, \mathrm{H}_{3}\right), 7.25\left(\mathrm{~s}, 1 \mathrm{H}, \mathrm{H}_{13}\right), 7.72$ $\left(\mathrm{s}, 1 \mathrm{H}, \mathrm{H}_{10}\right) ; \delta_{\mathrm{C}} 56.1\left(\mathrm{OCH}_{3}(1)\right.$ and $\left.(2)\right), 68.1\left(\mathrm{C}_{7}\right), 109.7\left(\mathrm{C}_{13}\right)$, $118.9\left(\mathrm{C}_{8}\right), 119.5\left(\mathrm{C}_{6}\right), 121.5\left(\mathrm{C}_{4}\right), 122.1\left(\mathrm{C}_{2}\right), 122.3\left(\mathrm{C}_{10}\right), 126.4$ $\left(\mathrm{C}_{3 / 5}\right), 136.9\left(\mathrm{C}_{9}\right), 139.3\left(\mathrm{C}_{11}\right), 145.9\left(\mathrm{C}_{12}\right), 148.1\left(\mathrm{C}_{1}\right) ; \mathrm{m} / \mathrm{z} 196$, $305[\mathrm{M}+\mathrm{H}]^{+}$. HRMS, calculated: $\mathrm{m} / \mathrm{z}$ 305.1093, found: $\mathrm{m} / \mathrm{z}$ $305.1131\left([\mathrm{M}+\mathrm{H}]^{+}\right)$.

2-((4,5-Dimethoxy-2-nitrobenzyl)oxy)-N-methylaniline (14). The aniline 14 was obtained after purification by column chromatography on silica gel (15\% EtOAc/cyclohexane) as an orange solid (60 mg, 48\%); rf 0.36 (20\% EtOAc/cyclohexane); mp $130{ }^{\circ} \mathrm{C}$; IR $\left(\mathrm{cm}^{-1}\right)$ 2976, 2999, 1215; $\delta_{\mathrm{H}} 2.9\left(\mathrm{~s}, 3 \mathrm{H}, \mathrm{NCH}_{3}\right), 3.90$ (s, 3H, $\left.\mathrm{OCH}_{3}(1)\right), 3.95$ (s, 3H, $\left.\mathrm{OCH}_{3}(2)\right), 4.32$ (bs, $\left.1 \mathrm{H}, \mathrm{NH}\right)$, $5.51\left(\mathrm{~s}, 2 \mathrm{H}, \mathrm{H}_{7}\right), 6.58\left(\mathrm{~d}, 1 \mathrm{H}, J=1.32 \mathrm{~Hz}, \mathrm{H}_{5}\right), 6.61(\mathrm{~d}, 1 \mathrm{H}, J=$ $\left.1.32 \mathrm{~Hz}, \mathrm{H}_{4}\right), 6.65\left(\mathrm{~d}, 1 \mathrm{H}, J=7.62 \mathrm{~Hz}, \mathrm{H}_{6}\right), 6.93(\mathrm{~d}, 1 \mathrm{H}, J=$ $\left.7.89 \mathrm{~Hz}, \mathrm{H}_{3}\right), 7.21\left(\mathrm{~s}, 1 \mathrm{H}, \mathrm{H}_{13}\right), 7.44\left(\mathrm{~s}, 1 \mathrm{H}, \mathrm{H}_{10}\right) ; \delta_{\mathrm{C}} 30.5$ $\left(\mathrm{NCH}_{3}\right), 56.4\left(\mathrm{OCH}_{3}(1) \&(2)\right), 68.1\left(\mathrm{C}_{7}\right), 109.7\left(\mathrm{C}_{13}\right), 118.9\left(\mathrm{C}_{8}\right)$, $119.5\left(\mathrm{C}_{6}\right), 121.5\left(\mathrm{C}_{4}\right), 122.1\left(\mathrm{C}_{2}\right), 122.3\left(\mathrm{C}_{11}\right), 126.4\left(\mathrm{C}_{3 / 5}\right), 136.9$ $\left(\mathrm{C}_{9}\right), 139.3\left(\mathrm{C}_{11}\right), 145.9\left(\mathrm{C}_{12}\right), 148.1\left(\mathrm{C}_{1}\right) ; \mathrm{m} / z$ 196, $319[\mathrm{M}+\mathrm{H}]^{+}$. 
HRMS, calculated: $m / z$ 319.1249, found: $\mathrm{m} / z \quad 319.1288$ $\left([\mathrm{M}+\mathrm{H}]^{+}\right)$.

6,8-Dichloro-9,9-dimethyl-7-oxo-7,9-dihydroacridin-2-yl(2-((4,5dimethoxy-2-nitrobenzyl)oxy)phenyl)(methyl)carbamate $\left(\mathbf{2}-\mathbf{H P C}^{\boldsymbol{H}}\right)$. The crude product was purified by column chromatography on silica gel ( $100 \%$ dichloromethane) to give the carbamate 2-HPC ${ }^{\mathbf{H}}$ (110.4 mg, quantitative yield) as a yellow solid; rf 0.27 (20\% EtOAc/cyclohexane); $\mathrm{mp} 160{ }^{\circ} \mathrm{C}$; IR $\left(\mathrm{cm}^{-1}\right)$ 3382, 2915, 2850, 2813, 1710, 1502; $\delta_{\mathrm{H}} 1.58$ (s, 6H, $\mathrm{CH}_{3}$ DDAO), 3.32 $\left(\mathrm{s}, 3 \mathrm{H}, \mathrm{NCH}_{3}\right), 3.82\left(\mathrm{~s}, 3 \mathrm{H}, \mathrm{OCH}_{3}(1)\right), 3.95\left(\mathrm{~s}, 3 \mathrm{H}, \mathrm{OCH}_{3}(2)\right)$, $5.52\left(\mathrm{~s}, 2 \mathrm{H}, \mathrm{H}_{7}\right), 6.58\left(\mathrm{~d}, 1 \mathrm{H}, J=1.32 \mathrm{~Hz}, \mathrm{H}_{5}\right), 6.61(\mathrm{~d}, 1 \mathrm{H}, J=$ $\left.1.32 \mathrm{~Hz}, \mathrm{H}_{4}\right), 6.75$ (d, $\left.1 \mathrm{H}, J=7.62 \mathrm{~Hz}, \mathrm{H}_{6}\right), 6.87\left(\mathrm{~s}, 1 \mathrm{H}, \mathrm{H}_{22}\right.$ ), 6.93 (d, 1H, J = $\left.7.89 \mathrm{~Hz}, \mathrm{H}_{3}\right), 7.11$ (s, 1H, H ${ }_{26}$ ), 7.21 (s, 1H, $\mathrm{H}_{13}$ ), $7.22\left(\mathrm{~s}, 1 \mathrm{H}, \mathrm{H}_{25}\right), 7.38\left(\mathrm{~s}, 1 \mathrm{H}, \mathrm{H}_{15}\right), 7.44\left(\mathrm{~s}, 1 \mathrm{H}, \mathrm{H}_{10}\right) ; \delta_{\mathrm{C}} 27.8$ $\left(\mathrm{CH}_{3} \mathrm{DDAO}\right), 30.5\left(\mathrm{NCH}_{3}\right), 32.8\left(\mathrm{C}_{17}\right), 56.4\left(\mathrm{OCH}_{3}(1)\right.$ \& (2)), $68.1\left(\mathrm{C}_{7}\right), 109.7\left(\mathrm{C}_{13}\right), 118.8\left(\mathrm{C}_{15}\right), 118.9\left(\mathrm{C}_{8}\right), 119.5\left(\mathrm{C}_{6}\right), 120.1$ $\left(\mathrm{C}_{26}\right), 120.4\left(\mathrm{C}_{25}\right), 121.5\left(\mathrm{C}_{4}\right), 122.1\left(\mathrm{C}_{2}\right), 122.3\left(\mathrm{C}_{10}\right), 126.4$ $\left(\mathrm{C}_{3 / 5}\right), 129.4\left(\mathrm{C}_{21}\right), 129.9\left(\mathrm{C}_{19}\right), 136.9\left(\mathrm{C}_{9}\right), 139.3\left(\mathrm{C}_{11}\right), 140.8$ $\left(\mathrm{C}_{24}\right), 144.6\left(\mathrm{C}_{16}\right), 145.9\left(\mathrm{C}_{12}\right), 147.2\left(\mathrm{C}_{18}\right), 148.1\left(\mathrm{C}_{1}\right), 151.2$ $\left(\mathrm{C}_{22}\right), 158.8\left(\mathrm{C}_{14}\right), 164.6\left(\mathrm{C}_{23}\right), 170.2\left(\mathrm{C}_{20}\right) ; \mathrm{m} / \mathrm{z}$ 196, 345, 652 $[\mathrm{M}+\mathrm{H}]^{+}$. HRMS, calculated: $\mathrm{m} / \mathrm{z}$ 652.1146, found: $\mathrm{m} / \mathrm{z}$ $652.1252\left([\mathrm{M}+\mathrm{H}]^{+}\right), 654.1235\left(\left[\mathrm{MCl}^{37}+\mathrm{H}\right]^{+}\right)$.

tert-Butyl(2-hydroxy-5-methoxyphenyl)carbamate (3). The carbamate 3 was obtained as a dark red oil $(555.1 \mathrm{mg}$, quantitative); rf 0.77 (40\% EtOAc/cyclohexane); IR $\left(\mathrm{cm}^{-1}\right)$ 3225, 2857, 1726,$1150 ; \delta_{\mathrm{H}} 3.66$ (s, 3H, methoxy), 6.14 (dd, $1 \mathrm{H}, J=2.94 / 8.94$ $\left.\mathrm{Hz}, \mathrm{H}_{5}\right), 6.36\left(\mathrm{~d}, 1 \mathrm{H}, J=2.91 \mathrm{~Hz}, \mathrm{H}_{3}\right), 6.58(\mathrm{~d}, 1 \mathrm{H}, J=8.58 \mathrm{~Hz}$, $\left.\mathrm{H}_{6}\right) ; \delta_{\mathrm{C}} 55.6\left(\mathrm{OCH}_{3}\right.$ phenol), $106.3\left(\mathrm{C}_{3}\right), 110\left(\mathrm{C}_{6}\right), 118.2\left(\mathrm{C}_{5}\right)$, $126.3\left(\mathrm{C}_{2}\right), 140.3\left(\mathrm{C}_{1}\right), 153.5\left(\mathrm{C}_{4}\right) ; \mathrm{m} / z 140[\mathrm{M}+\mathrm{H}]^{+}, 206,262$ $[\mathrm{M}+\mathrm{Na}]^{+}$. HRMS, calculated: $\mathrm{m} / \mathrm{z}$ 262.1091, found: $\mathrm{m} / \mathrm{z}$ $262.1050\left([\mathrm{M}+\mathrm{Na}]^{+}\right)$.

tert-Butyl(2-((4,5-dimethoxy-2-nitrobenzyl)oxy)-5-methoxyphenyl) carbamate (7). The crude product was purified by column chromatography on silica gel (50\% dichloromethane/cyclohexane) to give the ether 7 as a red solid (485.5 mg, 71\%); rf 0.33 (20\% EtOAc/cyclohexane); $\mathrm{mp} 143{ }^{\circ} \mathrm{C}$; IR $\left(\mathrm{cm}^{-1}\right) 3451$, 2975, 2904, 2863, 1712, 1271; $\delta_{\mathrm{H}} 1.49(\mathrm{~s}, 9 \mathrm{H}, t \mathrm{Bu}), 3.68(\mathrm{~s}, 3 \mathrm{H}$, methoxy), 6.47 (dd, $1 \mathrm{H}, J=2.88 / 8.70 \mathrm{~Hz}, \mathrm{H}_{5}$ ), 6.78 (d, $1 \mathrm{H}, J=$ $\left.8,5 \mathrm{~Hz}, \mathrm{H}_{6}\right), 7.12\left(\mathrm{~d}, 1 \mathrm{H}, J=2.6 \mathrm{~Hz}, \mathrm{H}_{3}\right) ; \delta_{\mathrm{C}} 28.3\left(\mathrm{CH}_{3} \mathrm{Boc}\right), 55.6$ (methoxy), 81.8 (Cq Boc), $106.5\left(\mathrm{C}_{3}\right), 110.2\left(\mathrm{C}_{6}\right), 118.4\left(\mathrm{C}_{5}\right)$, $126.5\left(\mathrm{C}_{2}\right), 140.5\left(\mathrm{C}_{1}\right), 152.5(\mathrm{C}=\mathrm{O}$ Boc $), 153.7\left(\mathrm{C}_{4}\right) ; \mathrm{m} / \mathrm{z}$ 140, 196, 335, $379[\mathrm{M}+\mathrm{H}]^{+}, 457[\mathrm{M}+\mathrm{Na}]^{+}$. HRMS, calculated: $m / z$ 435.1789, found: $m / z 435.1763\left([\mathrm{M}+\mathrm{H}]^{+}\right)$.

2-((4,5-Dimethoxy-2-nitrobenzyl)oxy)-5-methoxyaniline

(11). The aniline 11 was obtained as a red solid $(m=639 \mathrm{mg}$, quantitative yield); rf 0.35 (30\% EtOAc/cyclohexane); mp $117{ }^{\circ} \mathrm{C}$; IR $\left(\mathrm{cm}^{-1}\right)$ 3193, 2918, 2863, 1208; $\delta_{\mathrm{H}} 3.67(\mathrm{~s}, 1 \mathrm{H}$, methoxy), 3.82 (s, $1 \mathrm{H}, \mathrm{OCH}_{3}$ (1)), 3.91 (s, 1H, $\mathrm{OCH}_{3}$ (2)), 5.4 $\left(\mathrm{s}, 2 \mathrm{H}, \mathrm{H}_{7}\right), 6.78$ (dd, $\left.1 \mathrm{H}, J=2.3 / 8.9 \mathrm{~Hz}, \mathrm{H}_{5}\right), 6.85$ (d, $1 \mathrm{H}, 8.9$ $\mathrm{Hz}, \mathrm{H}_{6}$ ), 6.99 (d, 1H, $2.3 \mathrm{~Hz}, \mathrm{H}_{3}$ ), 7.12 (s, 1H, H $\mathrm{H}_{13}$ ), 7.65 (s, 1H, $\mathrm{H}_{10}$ ), 11.08 (bs, 2H, $\left.\mathrm{NH}_{2}\right) ; \delta_{\mathrm{C}} 55.5$ (methoxy), $56.5\left(\mathrm{OCH}_{3}\right.$ (1) and (2)), $68.7\left(\mathrm{C}_{7}\right), 102.3\left(\mathrm{C}_{3}\right), 102.5\left(\mathrm{C}_{5}\right), 108\left(\mathrm{C}_{6}\right), 109.5\left(\mathrm{C}_{8}\right)$, $114.3\left(\mathrm{C}_{10} / \mathrm{C}_{13}\right), 129.9\left(\mathrm{C}_{2}\right), 139.2\left(\mathrm{C}_{4}\right), 140.2\left(\mathrm{C}_{1}\right), 148.8\left(\mathrm{C}_{9}\right)$, $153.9\left(\mathrm{C}_{12}\right), 155.2\left(\mathrm{C}_{11}\right) ; m / z$ 154, 182, 196, 335, $357[\mathrm{M}+\mathrm{H}]^{+}$. HRMS, calculated: $\mathrm{m} / \mathrm{z}$ 335.1165, found: $\mathrm{m} / \mathrm{z} 335.1239$ $\left([\mathrm{M}+\mathrm{H}]^{+}\right)$.
2-((4,5-Dimethoxy-2-nitrobenzyl)oxy)-5-methoxy-N-methylaniline (15). The aniline 15 was obtained after purification by column chromatography on silica gel (15\% EtOAc/cyclohexane) as a yellow solid (148.9 mg, 45\%); rf 0.51 (20\% EtOAc/cyclohexane); mp $153{ }^{\circ} \mathrm{C}$; IR $\left(\mathrm{cm}^{-1}\right)$ 3406, 2929, 1516; $\delta_{\mathrm{H}} 3.87\left(\mathrm{~s}, 3 \mathrm{H}, \mathrm{NCH}_{3}\right)$, 3.76 (s, 3H, methoxy), 3.92 (s, 3H, $\mathrm{OCH}_{3}$ (1)), 3.96 (s, 3H, $\mathrm{OCH}_{3}$ (2)), 5.46 (s, 2H, $\mathrm{H}_{7}$ ), 6.1 (dd, $\left.1 \mathrm{H}, J=3.00 / 9.00 \mathrm{~Hz}, \mathrm{H}_{5}\right), 6.24$ (d, $\left.1 \mathrm{H}, J=2.8 \mathrm{~Hz}, \mathrm{H}_{3}\right), 6.67\left(\mathrm{~d}, 1 \mathrm{H}, J=8.9 \mathrm{~Hz}, \mathrm{H}_{6}\right), 7.24(\mathrm{~s}, 1 \mathrm{H}$, $\left.\mathrm{H}_{13}\right), 7.75\left(\mathrm{~s}, 1 \mathrm{H}, \mathrm{H}_{10}\right) ; \delta_{\mathrm{C}} 30.3\left(\mathrm{NCH}_{3}\right), 56.1$ (methoxy), 56.4 $\left(\mathrm{OCH}_{3}\right.$ (1) \& (2)), $70.1\left(\mathrm{C}_{7}\right), 107\left(\mathrm{C}_{3}\right), 110.7\left(\mathrm{C}_{6}\right), 113.4\left(\mathrm{C}_{13}\right)$, $118.9\left(\mathrm{C}_{5}\right), 127\left(\mathrm{C}_{2}\right), 127.8\left(\mathrm{C}_{8}\right), 129.9\left(\mathrm{C}_{10}\right), 140.4\left(\mathrm{C}_{9}\right), 140.9$ $\left(\mathrm{C}_{1}\right), 142.4\left(\mathrm{C}_{11}\right), 154.2\left(\mathrm{C}_{4}\right), 156.6\left(\mathrm{C}_{12}\right) ; \mathrm{m} / z$ 152, $349[\mathrm{M}+\mathrm{H}]^{+}$. HRMS, calculated: $\mathrm{m} / \mathrm{z}$ 349.1355, found: $\mathrm{m} / \mathrm{z} 349.1393$ $\left([\mathrm{M}+\mathrm{H}]^{+}\right)$.

6,8-Dichloro-9,9-dimethyl-7-oxo-7,9-dihydroacridin-2-yl(2-((4,5dimethoxy-2-nitrobenzyl)oxy)-5-methoxyphenyl)(methyl)carbamate

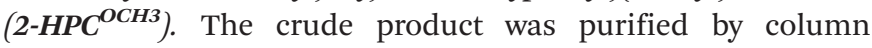
chromatography on silica gel (100\% dichloromethane) to give the final carbamate $2-\mathbf{H P C}{ }^{\mathbf{O C H}}$ ( $44.4 \mathrm{mg}$, quantitative yield) as a brown solid; rf 0.27 (20\% EtOAc/cyclohexane); mp $103{ }^{\circ} \mathrm{C}$; IR $\left(\mathrm{cm}^{-1}\right) 2924,2801,1726,1657,1235 ; \delta_{\mathrm{H}} 1.58\left(\mathrm{~s}, 6 \mathrm{H}, \mathrm{CH}_{3}\right.$ DDAO), 3.31 (s, 3H, $\mathrm{NCH}_{3}$ ), 3.77/3.81 (s, 6H, $\mathrm{OCH}_{3}$ (1) \& (2)), 3.91 (s, 3H, methoxy), 5.46 (s, 2H, $\left.\mathrm{H}_{7}\right), 6.87$ (m, 3H, $\left.\mathrm{H}_{3} / \mathrm{H}_{5} / \mathrm{H}_{6}\right)$, 6.97 (d, 1H, $\left.J=3 \mathrm{~Hz}, \mathrm{H}_{15}\right), 7.03$ (s, 1H, H $\left.\mathrm{H}_{25}\right), 7.38$ (s, 1H, $\mathrm{H}_{13}$ ), 7.44 (d, $\left.1 \mathrm{H}, J=9 \mathrm{~Hz}, \mathrm{H}_{26}\right), 7.51\left(\mathrm{~s}, 1 \mathrm{H}, \mathrm{H}_{22}\right), 7.74\left(\mathrm{~s}, 1 \mathrm{H}, \mathrm{H}_{10}\right)$; $\delta_{\mathrm{C}} 29.7\left(\mathrm{CH}_{3} \mathrm{DDAO}\right), 39.1\left(\mathrm{NCH}_{3}\right), 56.9 / 56.5 / 55.8\left(\mathrm{OCH}_{3}(1) \&\right.$ (2), methoxy), $67.7\left(\mathrm{C}_{7}\right), 106.6\left(\mathrm{C}_{3} / \mathrm{C}_{5}\right), 108\left(\mathrm{C}_{13}\right), 108.6\left(\mathrm{C}_{6}\right)$, $113.4\left(\mathrm{C}_{15}\right), 113.9\left(\mathrm{C}_{25}\right), 114.9\left(\mathrm{C}_{26}\right), 120\left(\mathrm{C}_{2}\right), 121.5\left(\mathrm{C}_{8}\right), 121.5$ $\left(\mathrm{C}_{9}\right), 129\left(\mathrm{C}_{21}\right), 132.1\left(\mathrm{C}_{19}\right), 132.9\left(\mathrm{C}_{10}\right), 135.4\left(\mathrm{C}_{16}\right), 137.2\left(\mathrm{C}_{9}\right)$, $138.2\left(\mathrm{C}_{16}\right), 138.6\left(\mathrm{C}_{11}\right), 139.2\left(\mathrm{C}_{12}\right), 139.4\left(\mathrm{C}_{1}\right), 140.3\left(\mathrm{C}_{18}\right)$, $147.4\left(\mathrm{C}_{14}\right), 148\left(\mathrm{C}_{22}\right), 149.6\left(\mathrm{C}_{23}\right), 154.1\left(\mathrm{C}_{4}\right), 154.4(\mathrm{C}=\mathrm{O}$ carbamate), 173.1 ( $\mathrm{C}=\mathrm{O}$ DDAO); $m / z$ 149, 448, $682[\mathrm{M}+\mathrm{H}]^{+}$. HRMS, calculated: $\mathrm{m} / \mathrm{z}$ 682.1251, found: $\mathrm{m} / \mathrm{z} 682.1356$ $\left([\mathrm{M}+\mathrm{H}]^{+}\right), 684.1341\left(\left[\mathrm{MCl}^{37}+\mathrm{H}\right]^{+}\right)$.

tert-Butyl(5-bromo-2-hydroxyphenyl)carbamate (4). The carbamate 4 was obtained as a red solid (750 mg, quantitative yield); rf 0.74 (40\% EtOAc/cyclohexane); mp $135{ }^{\circ} \mathrm{C}$; IR $\left(\mathrm{cm}^{-1}\right) 3262$, 2978, 1691, 1150; $\delta_{\mathrm{H}} 1.52(\mathrm{~s}, 9 \mathrm{H}, t \mathrm{Bu}), 6.65\left(\mathrm{~s}, 1 \mathrm{H}, \mathrm{H}_{3}\right), 6.82(\mathrm{~d}$, $\left.1 \mathrm{H}, J=8.58 \mathrm{~Hz}, \mathrm{H}_{6}\right), 7.11\left(\mathrm{dd}, 1 \mathrm{H}, J=3.87 / 8.49 \mathrm{~Hz}, \mathrm{H}_{5}\right), 7.33(\mathrm{~s}$, $1 \mathrm{H}, \mathrm{OH}), 7.87$ (bs, 1H, NH); $\delta_{\mathrm{C}} 28.2\left(\mathrm{CH}_{3} \mathrm{Boc}\right), 82.6$ (Cq Boc), $112.4\left(\mathrm{C}_{4}\right), 120\left(\mathrm{C}_{6}\right), 123.8\left(\mathrm{C}_{3}\right), 127.1\left(\mathrm{C}_{2}\right), 128.1\left(\mathrm{C}_{5}\right), 146.4$ $\left(\mathrm{C}_{1}\right), 154.6\left(\mathrm{C}=\mathrm{O}\right.$ Boc); $m / z$ 187, $231[\mathrm{M}+\mathrm{H}]^{+}, 310[\mathrm{M}+\mathrm{Na}]^{+}$. HRMS, calculated: $\mathrm{m} / \mathrm{z} 310.0037$, found: $\mathrm{m} / \mathrm{z} 310.0048$ $\left([\mathrm{M}+\mathrm{Na}]^{+}\right), 312.0029\left(\left[\mathrm{MBr}^{81}+\mathrm{Na}\right]^{+}\right)$.

tert-Butyl(5-bromo-2-((4,5-dimethoxy-2-nitrobenzyl)oxy)phenyl) carbamate (8). The crude product was purified by column chromatography on silica gel (20\% EtOAc/cyclohexane) to give the ether as a brown solid (1.23 g, 94\%); rf 0.34 (20\% EtOAc/ cyclohexane); mp $148{ }^{\circ} \mathrm{C}$; IR $\left(\mathrm{cm}^{-1}\right)$ 3452, 2976, 2927, 1726; $\delta_{\mathrm{H}} 1.52(\mathrm{~s}, 9 \mathrm{H}, t \mathrm{Bu}), 3.92\left(\mathrm{~s}, 3 \mathrm{H}, \mathrm{OCH}_{3}(1)\right), 3.97\left(\mathrm{~s}, 3 \mathrm{H}, \mathrm{OCH}_{3}\right.$ (2)), $5.53\left(\mathrm{~s}, 2 \mathrm{H}, \mathrm{H}_{7}\right), 6.69$ (d, $1 \mathrm{H}, J=8.70 \mathrm{~Hz}, \mathrm{H}_{6}$ ), 7.03 (dd, $1 \mathrm{H}$, $\left.J=2.31 / 8.61 \mathrm{~Hz}, \mathrm{H}_{5}\right), 7.11\left(\mathrm{~s}, 2 \mathrm{H}, \mathrm{H}_{3} / \mathrm{H}_{13}\right), 7.77\left(\mathrm{~s}, 1 \mathrm{H}, \mathrm{H}_{10}\right), 8.3$ (bs, $1 \mathrm{H}, \mathrm{NH}) ; \delta_{\mathrm{C}} 28.3(t \mathrm{Bu}), 56.4\left(\mathrm{OCH}_{3}(1) \&(2)\right), 68.3\left(\mathrm{C}_{7}\right), 81.1$ (Cq Boc), $108.2\left(\mathrm{C}_{13}\right), 109\left(\mathrm{C}_{4}\right), 113.3\left(\mathrm{C}_{6}\right), 114.7\left(\mathrm{C}_{3}\right), 121.4$ $\left(\mathrm{C}_{2}\right), 125.1\left(\mathrm{C}_{8}\right), 129.6\left(\mathrm{C}_{10}\right), 139.1\left(\mathrm{C}_{9}\right), 145.1\left(\mathrm{C}_{11}\right), 148.2\left(\mathrm{C}_{11}\right)$, $152.3\left(\mathrm{C}=\mathrm{O}\right.$ Boc), $154\left(\mathrm{C}_{12}\right) ; m / z$ 196, 385, 427, $483[\mathrm{M}+\mathrm{H}]^{+}$. 
HRMS, calculated: $m / z$ 483.0668, found: $m / z \quad 483.0766$ $\left([\mathrm{M}+\mathrm{H}]^{+}\right), 485.0738\left(\left[\mathrm{MBr}^{81}+\mathrm{H}\right]^{+}\right)$.

5-Bromo-2-((4,5-dimethoxy-2-nitrobenzyl)oxy)aniline (12). The aniline 18 was obtained as a dark red solid $(955.1 \mathrm{mg}$, quantitative yield); rf 0.23 (30\% EtOAc/cyclohexane); mp $142{ }^{\circ} \mathrm{C}$; IR $\left(\mathrm{cm}^{-1}\right)$ 3366, 2917, 2884, 1271, 1192; $\delta_{\mathrm{H}} 3.64$ (bs, 2H, $\left.\mathrm{NH}_{2}\right)$; 3.92 (s, $\left.3 \mathrm{H}, \mathrm{OCH}_{3}(1)\right) ; 3.95$ (s, 3H, $\left.\mathrm{OCH}_{3}(2)\right) ; 5,49$ (s, 2H, H $)$; $6,62\left(\mathrm{~d}, 1 \mathrm{H}, J=8.58 \mathrm{~Hz}, \mathrm{H}_{6}\right) ; 6.75\left(1 \mathrm{H}, \mathrm{dd}, 2.19 / 8.52 \mathrm{~Hz}, \mathrm{H}_{5}\right)$, $6.86\left(1 \mathrm{H}, \mathrm{d}, 2.22 \mathrm{~Hz}, \mathrm{H}_{3}\right), 7.17\left(1 \mathrm{H}, \mathrm{s}, \mathrm{H}_{13}\right), 7.74\left(1 \mathrm{H}, \mathrm{s}, \mathrm{H}_{10}\right)$; $\delta_{\mathrm{C}} 56.4\left(\mathrm{OCH}_{3}(1) \&(2)\right), 68.0\left(\mathrm{C}_{7}\right), 108.1\left(\mathrm{C}_{13}\right), 109.4\left(\mathrm{C}_{4}\right), 114.1$ $\left(\mathrm{C}_{3}\right), 114.4\left(\mathrm{C}_{6}\right), 118.0\left(\mathrm{C}_{5}\right), 121.0\left(\mathrm{C}_{8}\right), 128.9\left(\mathrm{C}_{10}\right), 138.0\left(\mathrm{C}_{2}\right)$, $139.3\left(\mathrm{C}_{9}\right), 144.7\left(\mathrm{C}_{11}\right), 148.0\left(\mathrm{C}_{1}\right), 153.9\left(\mathrm{C}_{12}\right) ; \mathrm{m} / \mathrm{z} 196,383$ $[\mathrm{M}+\mathrm{H}]^{+}$. HRMS, calculated: $\mathrm{m} / \mathrm{z}$ 383.0144, found: $\mathrm{m} / \mathrm{z}$ $383.0234\left([\mathrm{M}+\mathrm{H}]^{+}\right), 385.0217\left(\left[\mathrm{MBr}^{81}+\mathrm{H}\right]^{+}\right)$.

5-Bromo-2-((4,5-dimethoxy-2-nitrobenzyl)oxy)-N-methylaniline (16). The aniline 16 was obtained after purification by column chromatography on silica gel (15\% EtOAc/cyclohexane) as a yellow solid (496 mg, 50\%); rf 0.26 (20\% EtOAc/cyclohexane); mp $170{ }^{\circ} \mathrm{C}$; IR $\left(\mathrm{cm}^{-1}\right)$ 2922, 28.47, 1209, 1155; $\delta_{\mathrm{H}} 2.88(3 \mathrm{H}, \mathrm{s}$, $\left.\mathrm{NCH}_{3}\right), 3.91\left(3 \mathrm{H}, \mathrm{s}, \mathrm{OCH}_{3}(1)\right), 3.96\left(3 \mathrm{H}, \mathrm{s}, \mathrm{OCH}_{3}(2)\right), 5.50(2 \mathrm{H}$, $\left.\mathrm{s}, \mathrm{H}_{7}\right), 6.60\left(1 \mathrm{H}, \mathrm{d}, J=8.46 \mathrm{~Hz}, \mathrm{H}_{6}\right), 6.76(1 \mathrm{H}, \mathrm{d}, J=8.31 \mathrm{~Hz}$, $\left.\mathrm{H}_{5}\right), 6.82\left(1 \mathrm{H}, \mathrm{s}, \mathrm{H}_{3}\right), 7.15\left(1 \mathrm{H}, \mathrm{s}, \mathrm{H}_{13}\right), 7.75\left(1 \mathrm{H}, \mathrm{s}, \mathrm{H}_{10}\right) ; \delta_{\mathrm{C}} 30.4$ $\left(\mathrm{NCH}_{3}\right), 56.5\left(\mathrm{OCH}_{3}(1) \&(2)\right), 68.3\left(\mathrm{C}_{7}\right), 108.0\left(\mathrm{C}_{13}\right), 109.5\left(\mathrm{C}_{3}\right)$, $113.0\left(\mathrm{C}_{4}\right), 115.4\left(\mathrm{C}_{6}\right), 119.1\left(\mathrm{C}_{5}\right), 129.0\left(\mathrm{C}_{8}\right), 139.4\left(\mathrm{C}_{10}\right)$, $140.6\left(\mathrm{C}_{9}\right), 144.5\left(\mathrm{C}_{2}\right), 148.3\left(\mathrm{C}_{11} / \mathrm{C}_{1}\right), 154.1\left(\mathrm{C}_{12}\right) ; \mathrm{m} / \mathrm{z} 196,397$ $[\mathrm{M}+\mathrm{H}]^{+}$. HRMS, calculated: $\mathrm{m} / \mathrm{z}$ 397.0300, found: $\mathrm{m} / \mathrm{z}$ $397.0392\left([\mathrm{M}+\mathrm{H}]^{+}\right), 399.0373\left(\left[\mathrm{MBr}^{81}+\mathrm{H}\right]^{+}\right)$.

6,8-Dichloro-9,9-dimethyl-7-oxo-7,9-dihydroacridin-2-yl(5-bromo2-((4,5-dimethoxy-2-nitrobenzyl)oxy)phenyl)(methyl)carbamate $\left(2-H P C^{B r}\right)$. The crude product was purified by column chromatography on silica gel (100\% dichloromethane) to give the carbamate $2-\mathbf{H P C}^{\mathbf{B r}}(24.1 \mathrm{mg}$, quantitative yield) as a dark green solid; rf 0.31 (20\% EtOAc/cyclohexane); mp $146{ }^{\circ} \mathrm{C}$; IR $\left(\mathrm{cm}^{-1}\right)$ 2923, 1728, 1658, 1222; $\delta_{\mathrm{H}} 1.67$ (s, 6H, $\left.\mathrm{CH}_{3} \mathrm{DDAO}\right), 3.38$ (s, 3H, $\mathrm{NCH}_{3}$ ), 3.88 (s, 3H, $\left.\mathrm{OCH}_{3}(1)\right), 3.99$ (s, 3H, $\left.\mathrm{OCH}_{3}(2)\right), 5.57$ $\left(\mathrm{s}, 2 \mathrm{H}, \mathrm{H}_{7}\right), 6.93\left(\mathrm{~d}, 1 \mathrm{H}, J=9 \mathrm{~Hz}, \mathrm{H}_{5}\right), 7.03$ (s, 1H, $\left.\mathrm{H}_{13}\right), 7.09$ (d, $\left.1 \mathrm{H}, J=6 \mathrm{~Hz}, \mathrm{H}_{3}\right), 7.39\left(\mathrm{~s}, 1 \mathrm{H}, \mathrm{H}_{6}\right), 7.52\left(\mathrm{~m}, 3 \mathrm{H}, \mathrm{H}_{25} / \mathrm{H}_{26} /\right.$ $\left.\mathrm{H}_{15}\right), 7.60\left(\mathrm{~s}, 1 \mathrm{H}, \mathrm{H}_{10}\right), 7.82\left(\mathrm{~s}, 1 \mathrm{H}, \mathrm{H}_{22}\right) ; \delta_{\mathrm{C}} 25.6 / 26.4\left(\mathrm{CH}_{3}\right.$ DDAO), $29.7\left(\mathrm{NCH}_{3}\right), 56.5\left(\mathrm{OCH}_{3}(1)\right), 56.9\left(\mathrm{OCH}_{3}(2)\right), 67.6$ $\left(\mathrm{C}_{17}\right), 68\left(\mathrm{C}_{7}\right), 108.2\left(\mathrm{C}_{13}\right), 108.4\left(\mathrm{C}_{4}\right), 113.5\left(\mathrm{C}_{6}\right), 114.7\left(\mathrm{C}_{15}\right)$, $119.8\left(\mathrm{C}_{26}\right), 121.4\left(\mathrm{C}_{25}\right), 128\left(\mathrm{C}_{3}\right), 131.6\left(\mathrm{C}_{8}\right), 132.3\left(\mathrm{C}_{5}\right), 132.7$ $\left(\mathrm{C}_{2}\right), 135.6\left(\mathrm{C}_{21}\right), 137.3\left(\mathrm{C}_{19}\right), 138.2\left(\mathrm{C}_{24}\right), 138.7\left(\mathrm{C}_{16}\right), 139.3$ $\left(\mathrm{C}_{11}\right), 140.2\left(\mathrm{C}_{12} / \mathrm{C}_{23}\right), 148.2\left(\mathrm{C}_{18}\right), 149.6\left(\mathrm{C}_{14} / \mathrm{C}_{22}\right), 152.6\left(\mathrm{C}_{1}\right)$, $153.9\left(\mathrm{C}_{9}\right), 154.4(\mathrm{C}=\mathrm{O}$ carbamate $), 173.1\left(\mathrm{C}_{20}\right) ; \mathrm{m} / \mathrm{z} 397$, $730[\mathrm{M}+\mathrm{H}]^{+}$. HRMS, calculated: $\mathrm{m} / \mathrm{z} 730.0260$, found: $\mathrm{m} / \mathrm{z}$ $730.0358\left([\mathrm{M}+\mathrm{H}]^{+}\right), 732.0333\left(\left[\mathrm{MBr}^{81}+\mathrm{H}\right]^{+}\right), \quad 734.0319$ $\left(\left[\mathrm{MCl}^{37} \mathrm{Br}^{81}+\mathrm{H}\right]^{+}\right)$.

4,4,5,7-Tetramethyl-2-chromanone (17). 3,5-Dimethylphenol (500 mg, $4.09 \mathrm{mmol}$ ) and 3,3-dimethylacrylic acid (409.5 mg, $4.09 \mathrm{mmol})$ were dissolved in toluene $(15 \mathrm{~mL})$. Then methyl sulfonic acid was added $(2.92 \mathrm{~mL}, 20.45 \mathrm{mmol})$. The mixture was stirred for $4 \mathrm{~h}$ under argon at $85^{\circ} \mathrm{C}$. After solvent evaporation, the reaction mixture was diluted with EtOAc and washed with $\mathrm{K}_{2} \mathrm{CO}_{3}(1 \mathrm{M})$ solution, water and brine; and finally dried over anhydrous magnesium sulphate. The solvent was removed under vacuum affording a yellow oil (827 mg, 99\%); rf
0.72 (30\% EtOAc/cyclohexane); IR $\left(\mathrm{cm}^{-1}\right)$ 2966, 1766; $\delta_{\mathrm{H}} 1.42$ $\left(6 \mathrm{H}, \mathrm{s}, \mathrm{H}_{10} / \mathrm{H}_{11}\right), 2.25\left(3 \mathrm{H}, \mathrm{s}, \mathrm{H}_{7}\right), 2.45\left(3 \mathrm{H}, \mathrm{s}, \mathrm{H}_{8}\right), 2.56(2 \mathrm{H}, \mathrm{s}$, $\mathrm{H}_{12}$ ), $6.72\left(2 \mathrm{H}, \mathrm{d}, J=4.86 \mathrm{~Hz}, \mathrm{H}_{4} / \mathrm{H}_{6}\right) ; \delta_{\mathrm{C}} 20.9\left(\mathrm{C}_{7}\right), 23.9\left(\mathrm{C}_{8}\right)$, $28.2\left(\mathrm{C}_{10} / \mathrm{C}_{11}\right), 35.5\left(\mathrm{C}_{9}\right), 46.0\left(\mathrm{C}_{12}\right), 117.0\left(\mathrm{C}_{6}\right), 126.9\left(\mathrm{C}_{4}\right), 130.1$ $\left(\mathrm{C}_{3}\right), 136.6\left(\mathrm{C}_{2}\right), 138.2\left(\mathrm{C}_{5}\right), 152.0\left(\mathrm{C}_{1}\right), 169.3\left(\mathrm{C}_{13}\right) ; \mathrm{m} / \mathrm{z} 175,205$ $[\mathrm{M}+\mathrm{H}]^{+}$. HRMS, calculated: $\mathrm{m} / \mathrm{z}$ 205.1184, found: $\mathrm{m} / \mathrm{z}$ $205.1222\left([\mathrm{M}+\mathrm{H}]^{+}\right)$.

2-(4-Hydroxy-2-methylbutan-2-yl)-3,5-dimethylphenol (18). To a solution of 4,4,5,7-tetramethyl-2-chromanone $(500 \mathrm{mg}$, $2.45 \mathrm{mmol})$ in anhydrous THF $(10 \mathrm{~mL})$ in an ice bath was added lithium aluminium hydride (766.6 $\mathrm{mg}, 12.12 \mathrm{mmol}$ ) portion wise. The heterogeneous mixture was allowed to warm at room temperature and stirred $1 \mathrm{~h} 30 \mathrm{~min}$ under argon. The excess of $\mathrm{LiAlH}_{4}$ was neutralized with $\mathrm{NH}_{4} \mathrm{Cl}$ at $0{ }^{\circ} \mathrm{C}$, and then the suspension was filtered on Celite 535; the resulting filtrate was diluted with EtOAc and treated with $1 \mathrm{M} \mathrm{HCl}$, water and brine; and finally dried on $\mathrm{MgSO}_{4}$, filtered and concentrated in vacuo. The crude product was purified by column chromatography on silica gel, giving the alcohol 18 as an off-white powder (479 mg, 94\%); rf 0.35 (30\% EtOAc/cyclohexane); mp $78{ }^{\circ} \mathrm{C}$; IR $\left(\mathrm{cm}^{-1}\right)$ 3508; $\delta_{\mathrm{H}}\left(\mathrm{CD}_{3} \mathrm{OD}\right) 1.55\left(6 \mathrm{H}, \mathrm{s}, \mathrm{H}_{10} / \mathrm{H}_{11}\right), 2.17$ $\left(3 \mathrm{H}, \mathrm{s}, \mathrm{H}_{7}\right), 2.27\left(2 \mathrm{H}, \mathrm{t}, J=6.24 \mathrm{~Hz}, \mathrm{H}_{12}\right), 2.48\left(3 \mathrm{H}, \mathrm{s}, \mathrm{H}_{8}\right), 3.62$ $\left(2 \mathrm{H}, \mathrm{dd}, J=7.24 / 14.61 \mathrm{~Hz}, \mathrm{H}_{13}\right), 6.34\left(1 \mathrm{H}, \mathrm{s}, \mathrm{H}_{4}\right), 6.45(1 \mathrm{H}, \mathrm{bs}$, $\mathrm{OH}), 6.49\left(1 \mathrm{H}, \mathrm{s}, \mathrm{H}_{6}\right) ; \delta_{\mathrm{C}}\left(\mathrm{CD}_{3} \mathrm{OD}\right) 20.6\left(\mathrm{C}_{7}\right), 26.0\left(\mathrm{C}_{8}\right), 32.1$ $\left(\mathrm{C}_{10} / \mathrm{C}_{11}\right), 45.2\left(\mathrm{C}_{12}\right), 61.9\left(\mathrm{C}_{13}\right), 113.6\left(\mathrm{C}_{6}\right), 127.2\left(\mathrm{C}_{4}\right)$, $136.7\left(\mathrm{C}_{2}\right), 138.4\left(\mathrm{C}_{3}\right), 139.9\left(\mathrm{C}_{5}\right), 156.1\left(\mathrm{C}_{1}\right) ; \mathrm{m} / z$ 123, 189, 209 $[\mathrm{M}+\mathrm{H}]^{+}, 231[\mathrm{M}+\mathrm{Na}]^{+}$. HRMS, calculated: $\mathrm{m} / z$ 209.1497, found: $m / z 209.1531\left([\mathrm{M}+\mathrm{H}]^{+}\right)$.

3-(2-((4,5-Dimethoxy-2-nitrobenzyl)oxy)-4,6-dimethylphenyl)-3methylbutan-1-ol (19). $\mathrm{K}_{2} \mathrm{CO}_{3}(559.8 \mathrm{mg}, 4.05 \mathrm{mmol}, 1.5$ eq.) was added to a solution of 2-(4-hydroxy-2-methylbutan-2-yl)3,5-dimethylphenol (563 mg, $2.7 \mathrm{mmol}, 1$ eq.) in DMF (12 mL). After $2 \mathrm{~min}, 4,5$-dimethoxy-2-nitrobenzyl bromide (740 mg, $2.7 \mathrm{mmol}, 1$ eq.) was added. The mixture was stirred under argon overnight, and then treated with an aqueous solution of $\mathrm{K}_{2} \mathrm{CO}_{3}$, water and brine; dried on $\mathrm{MgSO}_{4}$, filtered and evaporated. The crude product was purified by column chromatography on silica gel ( $40 \%$ EtOAc/cyclohexane) to give the ether as an orange solid (879 mg, 81\%); rf 0.17 (30\% EtOAc/cyclohexane); mp $86{ }^{\circ} \mathrm{C}$; IR $\left(\mathrm{cm}^{-1}\right) 3326,2969,1274 ; \delta_{\mathrm{H}} 1.58(6 \mathrm{H}, \mathrm{s}$, $\left.\mathrm{H}_{10} / \mathrm{H}_{11}\right), 2.21\left(3 \mathrm{H}, \mathrm{s}, \mathrm{H}_{7}\right), 2.24\left(2 \mathrm{H}, \mathrm{t}, J=7.24 \mathrm{~Hz}, \mathrm{H}_{12}\right), 2.51$ $\left(3 \mathrm{H}, \mathrm{s}, \mathrm{H}_{8}\right), 3.57\left(2 \mathrm{H}, \mathrm{t}, J=7.23 \mathrm{~Hz}, \mathrm{H}_{13}\right), 3.94\left(3 \mathrm{H}, \mathrm{s}, \mathrm{OCH}_{3}\right.$ (1)), 3.97 (3H, s, $\left.\mathrm{OCH}_{3}(2)\right), 5.50\left(2 \mathrm{H}, \mathrm{s}, \mathrm{H}_{14}\right), 6.58\left(2 \mathrm{H}, \mathrm{s}, \mathrm{H}_{4} /\right.$ $\left.\mathrm{H}_{6}\right), 7.37\left(1 \mathrm{H}, \mathrm{s}, \mathrm{H}_{20}\right), 7.78\left(1 \mathrm{H}, \mathrm{s}, \mathrm{H}_{17}\right) ; \delta_{\mathrm{C}} 21.0\left(\mathrm{C}_{7}\right), 26.2\left(\mathrm{C}_{8}\right)$, $32.7\left(\mathrm{C}_{10} / \mathrm{C}_{11}\right), 40.5\left(\mathrm{C}_{9}\right), 46.0\left(\mathrm{C}_{12}\right), 56.5\left(\mathrm{OCH}_{3}(1) \&(2)\right), 61.5$ $\left(\mathrm{C}_{13}\right), 69.4\left(\mathrm{C}_{14}\right), 108.5\left(\mathrm{C}_{20}\right), 110.6\left(\mathrm{C}_{6}\right), 114.0\left(\mathrm{C}_{4}\right), 129.0\left(\mathrm{C}_{15}\right)$, $130.6\left(\mathrm{C}_{2}\right), 131.4\left(\mathrm{C}_{17}\right), 137.1\left(\mathrm{C}_{3}\right), 138.3\left(\mathrm{C}_{5}\right), 139.3\left(\mathrm{C}_{16}\right), 148.5$ $\left(\mathrm{C}_{18}\right), 154.3\left(\mathrm{C}_{1}\right), 158.8\left(\mathrm{C}_{19}\right) ; \mathrm{m} / z$ 196, 318, 404, $421[\mathrm{M}+\mathrm{H}]^{+}$. HRMS, calculated: $\mathrm{m} / \mathrm{z}$ 404.2028, found: $\mathrm{m} / \mathrm{z} 404.2069$ $\left([\mathrm{M}+\mathrm{H}]^{+}\right)$.

3-(2-((4,5-Dimethoxy-2-nitrobenzyl)oxy)-4,6-dimethylphenyl)-3methylbutanal (20). To a solution of 3-(2-((4,5-dimethoxy-2nitrobenzyl)oxy)-4,6-dimethylphenyl)-3-methylbutan-1-ol (714.9 $\mathrm{mg}, 1.77 \mathrm{mmol}, 1 \mathrm{eq}$.$) in 10 \mathrm{~mL}$ of dichloromethane was added pyridinium dichromate (2.66 g, $7.08 \mathrm{mmol}, 4 \mathrm{eq}$.). The mixture was stirred under argon for $24 \mathrm{~h}$, then treated with $\mathrm{NH}_{4} \mathrm{Cl}$, 
water and brine; dried over $\mathrm{MgSO}_{4}$, filtered and concentrated in vacuo. The crude product was purified by column chromatography (20\% EtOAc/cyclohexane) to give the aldehyde as an orange solid (675 mg, 95\%); rf 0.51 (30\% EtOAc/cyclohexane); mp $106{ }^{\circ} \mathrm{C}$; IR $\left(\mathrm{cm}^{-1}\right)$ 2919, 1721, 1275; $\delta_{\mathrm{H}} 1.62\left(6 \mathrm{H}, \mathrm{s}, \mathrm{H}_{10} /\right.$ $\left.\mathrm{H}_{11}\right), 2.16\left(3 \mathrm{H}, \mathrm{s}, \mathrm{H}_{7}\right), 2.51\left(3 \mathrm{H}, \mathrm{s}, \mathrm{H}_{8}\right), 2.97\left(2 \mathrm{H}, \mathrm{s}, \mathrm{H}_{12}\right), 3.90$ $\left(3 \mathrm{H}, \mathrm{s}, \mathrm{OCH}_{3}(1)\right), 3.94\left(3 \mathrm{H}, \mathrm{s}, \mathrm{OCH}_{3}(2)\right), 5.47\left(2 \mathrm{H}, \mathrm{s}, \mathrm{H}_{14}\right), 6.52$ $\left(1 \mathrm{H}, \mathrm{s}, \mathrm{H}_{4}\right), 6.58\left(1 \mathrm{H}, \mathrm{s}, \mathrm{H}_{6}\right), 7.20\left(1 \mathrm{H}, \mathrm{s}, \mathrm{H}_{20}\right), 7.74\left(1 \mathrm{H}, \mathrm{s}, \mathrm{H}_{17}\right)$, $9.54\left(1 \mathrm{H}, \mathrm{s}, \mathrm{H}_{13}\right) ; \delta_{\mathrm{C}} 21.0\left(\mathrm{C}_{7}\right), 26.2\left(\mathrm{C}_{8}\right), 32.3\left(\mathrm{C}_{10} / \mathrm{C}_{11}\right), 39.2$ $\left(\mathrm{C}_{9}\right), 57.1\left(\mathrm{OCH}_{3}(1) \&(2)\right), 69.2\left(\mathrm{C}_{14}\right), 108.5\left(\mathrm{C}_{20}\right), 110.2\left(\mathrm{C}_{6}\right)$, $113.9\left(\mathrm{C}_{4}\right), 128.9\left(\mathrm{C}_{15}\right), 129.9\left(\mathrm{C}_{2}\right), 130.2\left(\mathrm{C}_{17}\right), 137.4\left(\mathrm{C}_{3}\right), 138.0$ $\left(\mathrm{C}_{5}\right), 139.3\left(\mathrm{C}_{12}\right), 148.4\left(\mathrm{C}_{18}\right), 154.4\left(\mathrm{C}_{1}\right), 157.8\left(\mathrm{C}_{19}\right), 204.1\left(\mathrm{C}_{13}\right)$; $\mathrm{m} / \mathrm{z}$ 196, 338, 384, 402, $419[\mathrm{M}+\mathrm{H}]^{+}$. HRMS, calculated: $\mathrm{m} / \mathrm{z}$ 402.1872, found: $m / z 402.1911\left([\mathrm{M}+\mathrm{H}]^{+}\right)$.

3-(2-((4,5-Dimethoxy-2-nitrobenzyl)oxy)-4,6-dimethylphenyl)-3methylbutanoic acid (21). 3-(2-((4,5-Dimethoxy-2-nitrobenzyl) oxy)-4,6-dimethylphenyl)-3-methylbutanal (116 mg, 0.29 $\mathrm{mmol}$ ) was dissolved in a $10 \mathrm{~mL}$ of acetone/tert-butanol/water $(17: 12: 3)$ mixture, then 2-methyl-2-butene (208 $\mu \mathrm{L}$, $1.96 \mathrm{mmol}, 6.75$ eq.), sodium chlorite $(137.7 \mathrm{mg}, 1.52 \mathrm{mmol}$, 5.25 eq.), and sodium dihydrogenophosphate (53 mg, $0.44 \mathrm{mmol}, 1.5$ eq.) were added. The mixture was stirred overnight, and then neutralized with $\mathrm{NH}_{4} \mathrm{Cl}$. The organic phase was diluted with EtOAc, treated with water and dried on $\mathrm{MgSO}_{4}$. After filtration, evaporation and purification on silica gel ( $40 \%$ EtOAc/cyclohexane), the acid was obtained as a light yellow powder (107.6 mg, 89\%); rf 0.40 (40\% EtOAc/cyclohexane); $\mathrm{mp} 120^{\circ} \mathrm{C}$; IR $\left(\mathrm{cm}^{-1}\right) 2927,1711,1275 ; \delta_{\mathrm{H}} 1.79(6 \mathrm{H}, \mathrm{s}$, $\left.\mathrm{H}_{10} \mathrm{H} / 11\right), 2.26\left(3 \mathrm{H}, \mathrm{s}, \mathrm{H}_{8}\right), 2.37\left(3 \mathrm{H}, \mathrm{s}, \mathrm{H}_{7}\right), 3.64\left(2 \mathrm{H}, \mathrm{s}, \mathrm{H}_{12}\right)$, $3.76\left(3 \mathrm{H}, \mathrm{s}, \mathrm{OCH}_{3}(1)\right), 3.90\left(3 \mathrm{H}, \mathrm{s}, \mathrm{OCH}_{3}(2)\right), 5.61\left(2 \mathrm{H}, \mathrm{s}, \mathrm{H}_{14}\right)$, $6.65\left(1 \mathrm{H}, \mathrm{s}, \mathrm{H}_{4}\right), 6.68\left(1 \mathrm{H}, \mathrm{s}, \mathrm{H}_{6}\right), 7.40\left(1 \mathrm{H}, \mathrm{s}, \mathrm{H}_{20}\right), 7.68(1 \mathrm{H}, \mathrm{s}$, $\left.\mathrm{H}_{17}\right) ; \delta_{\mathrm{C}} 19.2\left(\mathrm{C}_{7}\right), 21.4\left(\mathrm{C}_{8}\right), 26.0\left(\mathrm{C}_{9}\right), 32.3\left(\mathrm{C}_{10} / \mathrm{C}_{11}\right), 40.1\left(\mathrm{C}_{12}\right)$, $57.1\left(\mathrm{OCH}_{3}(1) \&(2)\right), 69.3\left(\mathrm{C}_{14}\right), 108.4\left(\mathrm{C}_{20}\right), 110.0\left(\mathrm{C}_{6}\right), 114.0$ $\left(\mathrm{C}_{4}\right), 128.8\left(\mathrm{C}_{15}\right), 130.4\left(\mathrm{C}_{2}\right), 130.7\left(\mathrm{C}_{17}\right), 136.6\left(\mathrm{C}_{3}\right), 138.2\left(\mathrm{C}_{5}\right)$, $139.2\left(\mathrm{C}_{16}\right), 148.3\left(\mathrm{C}_{18}\right), 154.3\left(\mathrm{C}_{1}\right), 158.1\left(\mathrm{C}_{19}\right), 177.7\left(\mathrm{C}_{13}\right) ; \mathrm{m} / \mathrm{z}$ 196, 318, $418[\mathrm{M}+\mathrm{H}]^{+}, 435\left[\mathrm{M}+\mathrm{NH}_{4}\right]^{+}$. HRMS, calculated: $\mathrm{m} / \mathrm{z}$ 418.1821, found: $m / z 418.1860\left([\mathrm{M}+\mathrm{H}]^{+}\right)$.

6, 8-Dichloro-9,9-dimethyl-7-oxo-7,9-dihydroacridin-2-yl-3-(2((4,5-dimethoxy-2-nitrobenzyl)oxy)-4,6-dimethylphenyl)-3-methylbutanoate (TML). To a solution of 3-(2-((4,5-dimethoxy-2-nitrobenzyl)oxy)-4,6-dimethylphenyl)-3-methylbutanoic acid (15 mg, $0.036 \mathrm{mmol}, 1$ eq.) in anhydrous THF $(5 \mathrm{~mL})$, was injected an excess of $30 \%$ phosgene in toluene $(300 \mu \mathrm{L})$ under an inert atmosphere. The mixture was stirred at room temperature for $45 \mathrm{~min}$. The remaining phosgene was eliminated by an argon flux; this solution was then added to a solution of 1,3-dichloro-7-hydroxy-9,9-dimethylacridin-2( $9 H)$-one (11.1 mg, $0.036 \mathrm{mmol})$, 4-dimethylaminopyridine $(4.4 \mathrm{mg}, 0.036 \mathrm{mmol})$ and an excess of triethylamine $(1 \mathrm{~mL})$ in THF $(5 \mathrm{~mL})$. The resulting solution was stirred under argon at room temperature overnight. The solvent was evaporated, then the residue was taken up in dichloromethane; the organic phase was washed with water and brine; dried on $\mathrm{MgSO}_{4}$, filtered and concentrated in vacuo. The ester was purified by HPLC using 80 to $90 \%$ gradient of $\mathrm{CH}_{3} \mathrm{CN} /$ water/0.1\% TFA, Waters XBridge ${ }^{\circledR}$ Prep C18 $5 \mu \mathrm{m}$ OBD $^{\mathrm{TM}} 30 \times 150 \mathrm{~mm}$ Column, to give the pure product as a deep dark green solid $(25 \mathrm{mg}$, quantitative yield); rf 0.40 (40\% EtOAc/cyclohexane); mp $220{ }^{\circ} \mathrm{C}$; IR $\left(\mathrm{cm}^{-1}\right) 2918,1754,1621 ; \delta_{\mathrm{H}} 1.71\left(\mathrm{~s}, 6 \mathrm{H}, \mathrm{CH}_{3} \mathrm{DDAO}\right), 2.21$ $\left(\mathrm{s}, 2 \mathrm{H}, \mathrm{H}_{7}\right), 2.57\left(\mathrm{~s}, 2 \mathrm{H}, \mathrm{H}_{8}\right), 3.09\left(\mathrm{~s}, 2 \mathrm{H}, \mathrm{H}_{19}\right), 4.01(\mathrm{~s}, 6 \mathrm{H}$, $\left.\mathrm{OCH}_{3}(1) \&(2)\right), 5.56\left(\mathrm{~s}, 2 \mathrm{H}, \mathrm{H}_{9}\right), 6.55-7.82(\mathrm{~m}, 7 \mathrm{H}, \mathrm{ArH}) ; \delta_{\mathrm{C}} 11$ $\left(\mathrm{C}_{7}\right), 14.1\left(\mathrm{C}_{8}\right), 20.7\left(\mathrm{C}_{16}\right), 23\left(\mathrm{C}_{17}\right), 23.7 / 25.8\left(\mathrm{CH}_{3} \mathrm{DDAO}\right), 38.9$ $\left(\mathrm{C}_{18}\right), 56.4\left(\mathrm{OCH}_{3} 1\right.$ and 2$), 68.2\left(\mathrm{C}_{9}\right), 70.1\left(\mathrm{C}_{19}\right), 108.1\left(\mathrm{C}_{2}\right)$, $109.7\left(\mathrm{C}_{3} / \mathrm{C}_{5}\right), 128.5\left(\mathrm{C}_{4}\right), 128.8\left(\mathrm{C}_{15} / \mathrm{C}_{23}\right), 129.8\left(\mathrm{C}_{10}\right), 130.9$ $\left(\mathrm{C}_{14} / \mathrm{C}_{13}\right), 132.4\left(\mathrm{C}_{6}\right), 147.9\left(\mathrm{C}_{1} / \mathrm{C}_{11}\right), 153.7\left(\mathrm{C}_{25} / \mathrm{C}_{27}\right), 167.8\left(\mathrm{C}_{26}\right)$, 187.8(C=O ester); $\mathrm{m} / \mathrm{z} 350,447,647,707[\mathrm{M}+\mathrm{H}]^{+}$. HRMS, calculated: $m / z$ 707.1819, found: $m / z 707.1922\left([\mathrm{M}+\mathrm{H}]^{+}\right)$, $709.1911\left(\left[\mathrm{MCl}^{37}+\mathrm{H}\right]^{+}\right)$.

\section{Analytical solutions}

All kinetic experiments have been performed in $\mathrm{CH}_{3} \mathrm{CN} / 0.1 \mathrm{M}$ Britton-Robinson buffer ${ }^{23} 1: 1(\mathrm{v}: \mathrm{v})$. All solutions were prepared using water purified through a Direct-Q 5 (Millipore, Billerica, MA).

UV-Visible absorption. UV/Vis absorption spectra were recorded in $1 \mathrm{~cm} \times 1 \mathrm{~cm}$ quartz cuvettes (Hellma) on a diode array UV/Vis spectrophotometer (Cary 300, Agilent, Thermo Scientific) at $298 \mathrm{~K}$.

\section{Steady-state fluorescence emission}

Corrected fluorescence spectra upon one-photon excitation were recorded with a Photon Technology International QuantaMaster QM-1 spectrofluorimeter (PTI, Monmouth Junction, NJ) equipped with a Peltier cell holder (TLC50, Quantum Northwest, Shoreline, WA). Solutions for fluorescence measurements were adjusted to $10 \mu \mathrm{M}$.

\section{Irradiation experiments}

One-photon irradiation experiments were carried out on the spectrofluorimeter. Irradiation was performed using a filtered $75 \mathrm{~W}$ xenon lamp at several slit widths on $400 \mu \mathrm{L}$ samples in $0.2 \times 1 \mathrm{~cm}^{2}$ quartz fluorescence cuvettes (Hellma) under constant stirring.

\section{Acknowledgements}

The ComUE Paris Sciences et Lettres (PSL) is acknowledged for financial support to SH. Christine Gaillet and Stéphanie Deville-Foillard are acknowledged for NMR measurements and HPLC, respectively.

\section{Notes and references}

1 P. L. Carl, P. K. Chakravaty and J. A. Katzellenbogen, J. Med. Chem., 1981, 24(5), 479-480.

2 A. Zheng, D. Shan and B. Wang, J. Org. Chem., 1999, 64, 156-161.

3 M. Shirangi, M. Najafi, D. T. S. Rijkers, R. J. Kok, W. E. Hennink and C. F. van Nostrum, Bioconjugate Chem., 2016, 27, 576-585. 
4 J. Sloniec-Myszk, U. Resch-Genger and A. Hennig, J. Phys. Chem. B, 2016, 120, 877-885.

5 A. B. Mauger, P. J. Burke, H. H. Somani, F. Friedlos and R. J. Knox, J. Med. Chem., 1994, 37, 3452-3458.

6 R. Madec-Lougerstay, J.-C. Florent and C. Monneret, J. Chem. Soc., Perkin Trans. 1, 1999, 1369-1375.

7 A. El Alaoui, N. Saha, F. Schmidt, C. Monneret and J.-C. Florent, Bioorg. Med. Chem., 2006, 14, 5012-5019.

8 Y.-L. Leu, C.-S. Chen, Y.-J. Wu and J.-W. Chern, J. Med. Chem., 2008, 51, 1740-1746.

9 A. Alouane, R. Labruère, T. Le Saux, F. Schmidt and L. Jullien, Angew. Chem., Int. Ed., 2015, 54, 7492-7509.

10 R. Labruère, A. Alouane, T. Le Saux, I. Aujard, P. Pelupessy, A. Gautier, S. Dubruille, F. Schmidt and L. Jullien, Angew. Chem., Int. Ed., 2012, 124, 9478-9481.

11 A. Alouane, R. Labruère, T. Le Saux, I. Aujard, S. Dubruille, F. Schmidt and L. Jullien, Chem. - Eur. J., 2013, 19, 1171711724.

12 A. Alouane, R. Labruère, K. J. Silvestre, T. Le Saux, F. Schmidt and L. Jullien, Chem. - Asian J., 2014, 9, 13341340 .
13 S. Chen, X. Zhao, J. Chen, J. Chen, L. Kuznetsova, S. S. Wong and I. Ojima, Bioconjugate Chem., 2010, 21, 979-987.

14 D. Shan, M. G. Nicolaou, R. T. Borchardt and B. Wang, J. Pharm. Sci., 1997, 86(7), 765-767.

15 S. Milstien and L. A. Cohen, J. Am. Chem. Soc., 1972, 94, 9158-9165.

16 R. B. Greenwald, Y. H. Choe, C. D. Conover, K. Shum, D. Wu and M. Royzen, J. Med. Chem., 2000, 43, 475-487.

17 S. S. Chandran, K. A. Dickson and R. T. Raines, J. Am. Chem. Soc., 2005, 127, 1652-1653.

18 M. N. Levine, L. D. Lavis and R. T. Raines, Molecules, 2008, 13, 204-211.

19 F. Schmidt, J.-C. Florent, C. Monneret, R. Straub, J. Czech, M. Gerken and K. Bosslet, Bioorg. Med. Chem. Lett., 1997, 7, 1071-1076.

20 M. N. Levine and R. T. Raines, Chem. Sci., 2012, 3, 2412.

21 M. A. DeWit and E. R. Gillies, Org. Biomol. Chem., 2011, 9, 1846-1854.

22 G. J. Atwell, B. M. Sykes, C. J. O’Connor and W. A. Denny, J. Med. Chem., 1994, 37, 371-380.

23 C. Frugoni, Gazz. Chim. Ital., 1957, 87, 403-407. 